\title{
Dynamic term-by-term stabilized finite element formulation using orthogonal subgrid-scales for the incompressible Navier-Stokes problem
}

\author{
E. Castillo ${ }^{\mathrm{a}}$ and R. Codina $\mathrm{b}^{\mathrm{b}, \mathrm{c}}$ \\ a Departamento de Ingeniería Mecánica, Universidad de Santiago de Chile, Av Libertador Bernardo O'Higgins \\ 3363, Santiago, Chile \\ ${ }^{b}$ Universitat Politècnica de Catalunya, Jordi Girona 1-3, Edifici C1, 08034, Barcelona, Spain \\ ${ }^{c}$ CIMNE - Centre Internacional de Metodes Numerics en Enginyeria, Gran Capità S/N 08034 Barcelona, Spain \\ ernesto.castillode@usach.cl (E. Castillo) and ramon.codina@upc.edu (R. Codina)
}

\begin{abstract}
In this paper, we propose and analyze the stability and the dissipative structure of a new dynamic term-byterm stabilized finite element formulation for the Navier-Stokes problem that can be viewed as a variational multiscale (VMS) method under some assumptions. The essential point of the formulation is the time dependent nature of the subscales and, contrary to residual-based formulations, the introduction of two velocity subscale components. They represent the components of the convective and the pressure gradient terms, respectively, of the momentum equation that cannot be captured by the finite element mesh. A key idea of the proposed method is that the convective subscale is close to a solenoidal field and the pressure gradient subscale is close to a potential field. The method ensures stability in anisotropic space-time discretizations, which is proved using numerical analysis for a linearized problem and demonstrated in classical numerical tests. The work includes a detailed description of the proposed formulation and several numerical examples that serve to justify our claims.
\end{abstract}

Keywords: Stabilized finite element methods, Variational Multiscale, Dynamic Subscales, Term-by-Term stabilization

\section{Introduction}

In the numerical simulation of physical phenomena many times anisotropic space-time discretization are needed. This anisotropy has to be understood as space-time partitions in which $h$ (space discretization size) and $\delta t$ (time step size of the time discretization) are independently refined, and in particular they do not need to satisfy the well know condition $h^{2} \leq C \delta t$, with $C$ a constant independent of $h$ and $\delta t$, that usually appears in stabilized finite element methods (see [1]).

The stabilized finite element methods that we will consider can be cast in the variational multiscale (VMS) framework [2] (see [3] for an overview). In particular, the unknown is split into its finite element component and the subgrid scale (or subscale), which cannot be captured by the finite element mesh. Approximating the subgrid scale in terms of the finite element scale provides a formulation that is (hopefully) stable for the latter. This is why the resulting methods can be termed as subgrid scale (SGS) stabilized finite element methods.

One of the most common approximations used in SGS stabilization methods is to neglect the time derivative of the subscales, which are then called quasi-static. The resulting formulations require the condition 
$h^{2} \leq C \delta t$ mentioned above, and are therefore unstable for anisotropic space-time discretizations $[4,5,6]$. This will not be the case in the formulation we present in this paper.

In [6], the precise definition of the numerical dissipation for the orthogonal subscale stabilization introduced in [4] for incompressible flows was given and analyzed in detail, comparing additionally the diffusion introduced by the numerical discretization of the problem with respect to the diffusion introduced by a classical large eddy simulation (LES) model. In [5], the role of the orthogonal projections was discussed and analyzed in detail, proving stability and opening the door to solve turbulent incompressible flows with arbitrary time step sizes for fixed meshes if dynamic SGS methods are used. Indeed, in the last years, some VMS finite element methods have been proved to be able to solve turbulent flows accurately, giving the possibility to represent the large scales and the inertial range of the Kolmogorov cascade [5, 6, 7, 8]. In these works, the tracking of the subscales in time required by the approximation of their time derivative has been proved to be crucial, and it has even been shown that it reduces the CPU time of the calculations due to the reduction of nonlinear iterations needed to solve each time step.

Stabilized finite element methods were initially motivated for the steady-state case and later extended to the transient case (see for example [9] for an early space-time approach). In a VMS method, the exact problem for the subgrid scales involves inverting a differential operator; the approximation to this operator leads to the stabilization parameters on which the formulation depends. A classical way to proceed is to consider the time derivative discretized by finite differences, and therefore to consider its effect as a source with coefficient $\delta t^{-1}$, and to neglect the time derivative of the subscales. Thus, the stabilization parameters depend on $\delta t^{-1}$. This in particular implies that the steady state solution, if it exists, depends on the value of $\delta t$ used to step in time. Apart from this inconsistency, as it has been mentioned this approach is unstable for anisotropic space-time discretizations, as pointed out in [10, 1].

The forcing term in the exact problem for the subscales contains essentially the residual of the finite element solution. When approximating the subscale, it is thus proportional to this residual, so that the resulting stabilized formulation is said to be residual-based. This is the most common option [2, 11, 12], leading to consistent problems for the finite element scale. In the VMS approach, the subscale is multiplied by the adjoint of the differential operator of the problem being solved applied to the test functions of the finite element scale, and the result integrated element-wise. We have observed that in some cases with non-smooth solutions, the cross products of the different components of the differential operator in the finite element residual and the adjoint operator applied to the test functions may yield local instabilities. In [13, 14], a non-residual-based VMS method for the momentum equation based on orthogonal projections was proved to be able to solve more elastic cases than a residual-based one. On the other hand, fractional step methods can be formulated more easily using non-residual-based methods due to the inclusion of less cross terms that do not play any stability role. Motivated in these two facts, we propose in this paper a dynamic term-by-term VMS method, with all the well known stability properties of the dynamic residual-based stabilized methods but with a term-by-term non-residual-based structure. The key ideas, explained along the paper, are the orthogonal projections that give place to an optimal order non-consistent method, and the tracking of the subscales in time. The novelty is that here we will consider two velocity subscales for incompressible flows.

The paper is organized as follows. In Section 2 the problem is stated. In Section 3 the stabilized finite element method we propose is presented, while in Section 4 the method is analyzed for a linearized problem. The numerical results used to test the new formulation are presented in Section 5, and, finally, conclusions 
are collected in Section 6 .

\section{Problem statement and Galerkin finite element discretization}

\subsection{Boundary value problem}

Let $\Omega \subset \mathbb{R}^{d}$, with $d=2$ or 3 , be the computational domain in which the flow takes place during the time interval $(0, T)$, assumed to be bounded and polyhedral, and let $\partial \Omega$ be its boundary. If $\boldsymbol{u}$ is the velocity field and $p$ the pressure, the incompressible Navier-Stokes equations for a Newtonian fluid are

$$
\begin{aligned}
\frac{\partial \boldsymbol{u}}{\partial t}+\boldsymbol{u} \cdot \nabla \boldsymbol{u}+\nabla p-\nu \Delta \boldsymbol{u} & =\boldsymbol{f}, & & \text { in } \Omega, t \in(0, T), \\
\nabla \cdot \boldsymbol{u} & =0, & & \text { in } \Omega, t \in(0, T),
\end{aligned}
$$

where $\boldsymbol{f}$ represents the vector of external forces and $\nu$ is the kinematic viscosity of the fluid. Equations (1) and (2) must be supplied with an initial condition of the form $\boldsymbol{u}=\boldsymbol{u}^{0}$ in $\Omega, t=0$, and a boundary condition which, for simplicity, will be taken as $\boldsymbol{u}=\mathbf{0}$ on $\partial \Omega, t \in(0, T)$.

\subsection{Dissipative structure}

In order to study the dissipative structure of the finite element method proposed later, we also consider the LES problem obtained by applying a filter of the form

$$
\overline{\boldsymbol{v}}(\boldsymbol{x})=\int \boldsymbol{v}\left(\boldsymbol{x}^{\prime}\right) G\left(\boldsymbol{x}, \boldsymbol{x}^{\prime}\right) d \boldsymbol{x}^{\prime},
$$

to the Navier-Stokes Equations (1) and (2) for an appropriate filter function $G\left(\boldsymbol{x}, \boldsymbol{x}^{\prime}\right)$, vanishing outside a ball of an appropriate diameter (the filter-width). The final filtered problem results in an extra term: the divergence of the residual stress tensor $\tau$, defined in components as $\tau_{i j}=\overline{u_{i} u_{j}}-\overline{u_{i}} \overline{u_{j}}$. The LES problem consist in finding the filtered velocity $(\overline{\boldsymbol{u}})$ and the filtered pressure $(\bar{p})$ as solution to the equations:

$$
\begin{aligned}
\frac{\partial \overline{\boldsymbol{u}}}{\partial t}+\overline{\boldsymbol{u}} \cdot \nabla \overline{\boldsymbol{u}}+\nabla \bar{p}-\nu \Delta \overline{\boldsymbol{u}}+\nabla \cdot \boldsymbol{\tau} & =\boldsymbol{f}, & \text { in } \Omega, t \in(0, T), \\
\nabla \cdot \overline{\boldsymbol{u}} & =0, & \text { in } \Omega, t \in(0, T) .
\end{aligned}
$$

A kinetic energy conservation statement for the Navier-Stokes problem can be found by multiplying the momentum Equation (1) by the velocity field $\boldsymbol{u}$. Taking into account the pointwise kinetic energy definition, $k=\frac{1}{2} \boldsymbol{u} \cdot \boldsymbol{u}$, and the divergence free nature of $\boldsymbol{u}$, the following equation is found:

$$
\frac{\partial k}{\partial t}+\boldsymbol{u} \cdot \nabla k+\boldsymbol{u} \cdot \nabla p-\nu \Delta k+\nu \nabla \boldsymbol{u}: \nabla \boldsymbol{u}=\boldsymbol{u} \cdot \boldsymbol{f}, \quad \text { in } \Omega, t \in(0, T),
$$

which integrating over an arbitrary volume $\omega \subset \Omega$ with exterior unit normal $\hat{\boldsymbol{n}}$ gives:

$$
\int_{\omega} \frac{\partial k}{\partial t}+\int_{\partial \omega} \hat{\boldsymbol{n}} \cdot[\boldsymbol{u}(k+p)-\nu \nabla k]+\int_{\omega} \nu \nabla \boldsymbol{u}: \nabla \boldsymbol{u}=\int_{\omega} \boldsymbol{u} \cdot \boldsymbol{f} .
$$

The total energy variation in volume $\omega$ (first term of the left-hand-side, LHS) is balanced by the flow of energy through its boundary (second term) plus the dissipation due to viscous effects (third term) and the work of external forces, given by the right-hand-side (RHS) term. 
A similar kinetic energy conservation equation is found multiplying the LES Equation (3) by the filtered velocity $\overline{\boldsymbol{u}}$. The result is:

$$
\frac{\partial \bar{k}}{\partial t}+\boldsymbol{u} \cdot \nabla \bar{k}+\overline{\boldsymbol{u}} \cdot \nabla \bar{p}-\nu \Delta \bar{k}+\nu \nabla \overline{\boldsymbol{u}}: \nabla \overline{\boldsymbol{u}}+\nabla \cdot(\overline{\boldsymbol{u}} \cdot \boldsymbol{\tau})+\nabla \overline{\boldsymbol{u}}: \boldsymbol{\tau}=\overline{\boldsymbol{u}} \cdot \boldsymbol{f} .
$$

This equation integrated over $\omega \in \Omega$ and simplified by the use of (4) gives:

$$
\int_{\omega} \frac{\partial \bar{k}}{\partial t}+\int_{\partial \omega} \hat{\boldsymbol{n}} \cdot[\overline{\boldsymbol{u}}(\bar{k}+\bar{p})-\nu \nabla \bar{k}+\overline{\boldsymbol{u}} \cdot \boldsymbol{\tau}]+\int_{\omega} \nu \nabla \overline{\boldsymbol{u}}: \nabla \overline{\boldsymbol{u}}+\int_{\omega} \nu \nabla \overline{\boldsymbol{u}}: \boldsymbol{\tau}=\int_{\omega} \overline{\boldsymbol{u}} \cdot \boldsymbol{f} .
$$

The interpretation of the terms corresponds to the meaning they had before, except for the last term of the LHS, that represents the energy transfer between coarse and fine scales, and the last component of the second term, which now includes the flow of the energy through the boundary due to the work done by the mean velocity against the residual stress tensor. Note that this term comes from the filtering of the convective term.

\subsection{Variational form}

From the point of view of the discrete approximation, the incompressible Navier-Stokes problem (1)(2), and the LES problem (3)-(4), are identical, except for the divergence of the residual stress tensor, and therefore the same discrete formulation is usually used.

Let us introduce some standard notation. The space of functions whose $l$-th power $(1 \leq l<\infty)$ is integrable in a domain $\omega$ is denoted by $L^{l}(\omega), L^{\infty}(\omega)$ being the space of bounded functions in $\omega$. The space of functions whose distributional derivatives of order up to $m \geq 0$ (integer) belong to $L^{2}(\omega)$ is denoted by $H^{m}(\omega)$. The space of functions in $H^{1}(\omega)$ vanishing on $\partial \Omega$ is denoted by $H_{0}^{1}(\Omega)$. The topological dual of $H_{0}^{1}(\omega)$ is denoted by $H^{-1}(\omega)$.

If $f$ and $g$ are functions (or distributions) such that $f g$ is integrable in the domain $\omega$ under consideration, we denote $\langle f, g\rangle_{\omega}=\int_{\omega} f g$, so that, in particular, $\langle\cdot, \cdot\rangle_{\omega}$ is the duality pairing between $H^{-1}(\omega)$ and $H_{0}^{1}(\omega)$. When $f, g \in L^{2}(\omega)$, we write the inner product as $\langle\cdot, \cdot\rangle_{\omega}=(\cdot, \cdot)_{\omega}$. This notation is simplified when $\omega=\Omega$ as follows: $\langle\cdot, \cdot\rangle_{\omega} \equiv\langle\cdot, \cdot\rangle$ and $(\cdot, \cdot)_{\omega} \equiv(\cdot, \cdot)$.

Let $\mathcal{V}=\left(H_{0}^{1}(\Omega)\right)^{d}$ and $\mathcal{Q}=L^{2}(\Omega) / \mathbb{R}$, the spaces of velocity and pressure, respectively. If we denote $\mathcal{X}:=\mathcal{V} \times \mathcal{Q}$, the weak form of the problem (1)-(2) consists in finding $\boldsymbol{U}=[\boldsymbol{u}, p]:(0, T) \rightarrow \mathcal{X}$ such that

$$
\left(\frac{\partial \boldsymbol{u}}{\partial t}, \boldsymbol{v}\right)+B(\boldsymbol{u} ; \boldsymbol{U}, \boldsymbol{V})=\langle\boldsymbol{f}, \boldsymbol{v}\rangle
$$

almost everywhere in $(0, T)$ and for all $\boldsymbol{V}=[\boldsymbol{v}, q] \in \mathcal{X}$, satisfying the initial condition in a weak $L^{2}$-sense, and with

$$
B(\boldsymbol{a} ; \boldsymbol{U}, \boldsymbol{V}):=\nu(\nabla \boldsymbol{u}, \nabla \boldsymbol{v})+\langle\boldsymbol{a} \cdot \nabla \boldsymbol{u}, \boldsymbol{v}\rangle-(p, \nabla \cdot \boldsymbol{v})+(q, \nabla \cdot \boldsymbol{u})
$$

where $\boldsymbol{f}$ has been assumed to belong to $\left(H^{-1}(\Omega)\right)^{d}$ for each $t \in(0, T)$.

\subsection{Galerkin finite element discretization and time discretization}

Let $\mathcal{T}_{h}=\{K\}$ be a finite element partition of the domain $\Omega$, considered polygonal for $d=2$ and polyhedral for $d=3$. For the sake of conciseness, we consider this partition quasi-uniform, of diameter $h$. From this we may construct conforming finite element spaces for the velocity and the pressure, $\mathcal{V}_{h} \subset \mathcal{V}$ and $\mathcal{Q}_{h} \subset \mathcal{Q}$, in 
the usual manner. The Galerkin finite element approximation consist in finding $\boldsymbol{U}_{h}=\left[\boldsymbol{u}_{h}, p_{h}\right]:(0, T) \rightarrow \mathcal{X}_{h}$, such that

$$
\left(\frac{\partial \boldsymbol{u}_{h}}{\partial t}, \boldsymbol{v}_{h}\right)+B\left(\boldsymbol{u}_{h} ; \boldsymbol{U}_{h}, \boldsymbol{V}_{h}\right)=\left\langle\boldsymbol{f}, \boldsymbol{v}_{h}\right\rangle,
$$

for all $\boldsymbol{V}_{h}=\left[\boldsymbol{v}_{h}, q_{h}\right] \in \mathcal{X}_{h}$, and satisfying the initial condition in a weak sense.

It is known that Equation (8) suffers from different types of numerical instabilities. On the one hand, if the convective term of the momentum equation dominates the viscous one, numerical oscillations may appear. On the other hand, the velocity-pressure spaces need to satisfy an inf-sup condition in order to have a well-posed problem. These difficulties are overcome by using a stabilized finite element method, as the one proposed in this work.

The term involving the time derivative can be discretized in different ways. However, the most common option consist in using finite difference schemes in time. In particular, we have implemented the first and the second order backward differencing schemes, based respectively on the approximations:

$$
\begin{aligned}
& \left.\frac{\partial \boldsymbol{u}_{h}}{\partial t}\right|_{t^{n+1}}=\frac{\boldsymbol{u}_{h}^{n+1}-\boldsymbol{u}_{h}^{n}}{\delta t}+\mathcal{O}(\delta t), \\
& \left.\frac{\partial \boldsymbol{u}_{h}}{\partial t}\right|_{t^{n+1}}=\frac{3 \boldsymbol{u}_{h}^{n+1}-4 \boldsymbol{u}_{h}^{n}+\boldsymbol{u}_{h}^{n-1}}{2 \delta t}+\mathcal{O}\left(\delta t^{2}\right),
\end{aligned}
$$

for $n=0,1,2, \ldots$ In both cases, $\delta t$ corresponds to the size of a uniform partition of the time interval $(0, T)$, while, $\mathcal{O}(\cdot)$ represents the approximation order of the scheme. The superscript indicates the time step where the variable is being approximated, so that $\boldsymbol{u}_{h}^{n}$ is an approximation to $\boldsymbol{u}_{h}$ at time $t^{n}=n \delta t$. The stabilized finite element method to be proposed next is independent of the time scheme used.

\section{Stabilized finite element formulation}

\subsection{Residual-based VMS methods}

The stabilized finite element formulation proposed in this work can be motivated in the framework of VMS methods and, in particular, in those method that model the SGS. The basic idea is to split the unknown $\boldsymbol{U}$ that belongs to $\mathcal{X}$ in two components, the finite element component $\boldsymbol{U}_{h}$ that belongs to the finite element space $\mathcal{X}_{h}$, and the remainder part, defined as the subscale component $\tilde{\boldsymbol{U}}$, that belongs to the subscale space $\tilde{\mathcal{X}}$, in such a way that $\boldsymbol{U}=\boldsymbol{U}_{h}+\tilde{\boldsymbol{U}}$ and $\mathcal{X}=\mathcal{X}_{h} \oplus \tilde{\mathcal{X}}$.

Let us consider a general nonlinear evolution problem of the form

$$
\boldsymbol{M}(\boldsymbol{U}) \frac{\partial \boldsymbol{U}}{\partial t}+\mathcal{L}(\boldsymbol{U}, \boldsymbol{U})=\boldsymbol{F},
$$

where $\boldsymbol{U}$ is the unknown of the problem, $\mathcal{L}(\boldsymbol{U}, \cdot)$ corresponds to a space differential operator, assumed to be linear in the second argument, $\boldsymbol{M}(\boldsymbol{U})$ is a matrix and $\boldsymbol{F}$ a force field. A general SGS method of VMS type can be derived in the way explained next.

First of all, let us state the weak form of the problem (11), which can be formally written as

$$
\left(\boldsymbol{M}(\boldsymbol{U}) \frac{\partial \boldsymbol{U}}{\partial t}, \boldsymbol{V}\right)+\langle\mathcal{L}(\boldsymbol{U}, \boldsymbol{U}), \boldsymbol{V}\rangle=\langle\boldsymbol{F}, \boldsymbol{V}\rangle,
$$

for an appropriate duality and considering $\boldsymbol{V}$ as a test function independent of time. The boundary conditions of the problem have to be taken into account, and are understood to be incorporated in the duality $\langle\cdot, \cdot\rangle$. 
The second step consists in splitting the weak form in two parts, the finite element and the subscale part, obtained by taking the test function in the respective spaces:

$$
\begin{array}{cc}
\left(\boldsymbol{M}(\boldsymbol{U}) \frac{\partial \boldsymbol{U}}{\partial t}, \boldsymbol{V}_{h}\right)+\left\langle\mathcal{L}(\boldsymbol{U}, \boldsymbol{U}), \boldsymbol{V}_{h}\right\rangle=\left\langle\boldsymbol{F}, \boldsymbol{V}_{h}\right\rangle & \forall \boldsymbol{V}_{h} \in \mathcal{X}_{h} \\
\left(\boldsymbol{M}(\boldsymbol{U}) \frac{\partial \boldsymbol{U}}{\partial t}, \tilde{\boldsymbol{V}}\right)+\langle\mathcal{L}(\boldsymbol{U}, \boldsymbol{U}), \tilde{\boldsymbol{V}}\rangle=\langle\boldsymbol{F}, \tilde{\boldsymbol{V}}\rangle & \forall \tilde{\boldsymbol{V}} \in \tilde{\mathcal{X}}
\end{array}
$$

This is exactly equivalent to the original problem (12). In essence, the goal of all SGS methods, including the approximation of the sub-scale with bubble functions, is to approximate $\tilde{U}$ in one way or another and end up with a problem for $\boldsymbol{U}_{h}$ alone, maintaining the number of unknowns of the Galerkin finite element problem.

In general, SGS methods have a residual-based structure, and therefore consistency comes from construction. Following the procedure described for example in [15], and after some simplifications and mathematical manipulations, the subscale component can be written in terms of the finite element part as:

$$
\boldsymbol{M}(\boldsymbol{U}) \frac{\partial \tilde{\boldsymbol{U}}}{\partial t}+\boldsymbol{\tau}^{-1}(\boldsymbol{U}) \tilde{\boldsymbol{U}}=\tilde{P}\left[\boldsymbol{F}-\boldsymbol{M}(\boldsymbol{U}) \frac{\partial \boldsymbol{U}_{h}}{\partial t}-\mathcal{L}\left(\boldsymbol{U}_{h}, \boldsymbol{U}_{h}\right)\right]
$$

where $\tau^{-1}(\cdot)$ is a matrix defined within each element domain that has to be determined, called matrix of stabilization parameters. Additionally, $\tilde{P}$ represents the $L^{2}$-projection onto the sub-scale space. Note that this projection should be applied also to the LHS; it would be the identity if $\boldsymbol{M}$ and $\boldsymbol{\tau}^{-1}$ were constants, so that omitting it has to be considered an approximation. Note also that the terms between brackets in the RHS represent the residual of the finite element approximation. If $\tilde{P}$ is taken as the projection onto the space of finite element residuals, we arrive to the most classical approach, the ASGS method (Algebraic Sub-Grid Scale method) [16]. If $\tilde{P}$ is taken as the orthogonal projection to the finite element space, we obtain the OSGS method (Orthogonal Sub-Grid Scale method) [16].

Applying the procedure described above to the linearized Navier-Stokes problem, with $\hat{\boldsymbol{u}}_{h}$ a given approximation of $\boldsymbol{u}$, we obtain the following residual-based stabilization method:

$$
\begin{aligned}
\left(\frac{\partial \boldsymbol{u}_{h}}{\partial t}, \boldsymbol{v}_{h}\right) & +B\left(\hat{\boldsymbol{u}}_{h} ; \boldsymbol{U}_{h}, \boldsymbol{V}_{h}\right)-\sum_{K}\left(\nabla \cdot \boldsymbol{v}_{h}, \tilde{p}\right)_{K} \\
& -\sum_{K}\left(\hat{\boldsymbol{u}}_{h} \cdot \nabla \boldsymbol{v}_{h}+\nu \Delta \boldsymbol{v}_{h}+\nabla q_{h}, \tilde{\boldsymbol{u}}\right)_{K}=\left\langle\boldsymbol{f}, \boldsymbol{v}_{h}\right\rangle,
\end{aligned}
$$

for all test functions $\boldsymbol{V}_{h}=\left[\boldsymbol{v}_{h}, q_{h}\right]$, where $\tilde{\boldsymbol{u}}$ and $\tilde{p}$, are the subscales of the momentum and continuity equation, respectively. In principle, $\hat{\boldsymbol{u}}_{h}=\boldsymbol{u}_{h}+\tilde{\boldsymbol{u}}$, but $\hat{\boldsymbol{u}}_{h} \approx \boldsymbol{u}_{h}$ leads also to a stable and accurate method (see [8] for the effects of the choice of $\hat{\boldsymbol{u}}_{h}$ in turbulence).

If we use a residual-based VMS method, the subscales are the solution of the problem:

$$
\begin{aligned}
\frac{\partial \tilde{\boldsymbol{u}}}{\partial t}+\tau_{1}^{-1} \tilde{\boldsymbol{u}} & =\tilde{P}\left(\boldsymbol{f}-\left(\frac{\partial \boldsymbol{u}_{h}}{\partial t}+\hat{\boldsymbol{u}}_{h} \cdot \nabla \boldsymbol{u}_{h}+\nabla p_{h}-\nu \Delta \boldsymbol{u}_{h}\right)\right), \\
\tau_{2}^{-1} \tilde{p} & =\tilde{P}\left(-\nabla \cdot \boldsymbol{u}_{h}\right),
\end{aligned}
$$

where $\boldsymbol{\tau}=\operatorname{diag}\left(\tau_{1} \boldsymbol{I}, \tau_{2}\right)$ are the components of the matrix of stabilization parameters, $\boldsymbol{I}$ being the $d \times d$ identity matrix. We will refer later to its structure. Note that the stabilization terms added to the Galerkin 
method in (16) are proportional to the finite element residuals of the momentum and the continuity equation. Throughout the work we will refer to the stabilized method defined by (16) as the residual-based VMS method, adding the prefix orthogonal if $\tilde{P}=P_{h}^{\perp}$.

If the time derivative of the velocity subscale in Equation (17) is neglected, we refer to the method as quasi-static; otherwise the method is called dynamic. It is understood that what is quasi-static or dynamic is the velocity subscale. When $\tilde{P}=P_{h}^{\perp}$, two additional simplifications can be done: $P_{h}^{\perp}\left(\frac{\partial \boldsymbol{u}_{h}}{\partial t}\right)=\mathbf{0}$, and $P_{h}^{\perp}(\boldsymbol{f}) \approx \mathbf{0}$; the latter yields a weakly consistent method (see below), and in fact it is exact if $\boldsymbol{f}$ is finite element function. The initial condition for the velocity subscale in (17) can be taken as zero (see [17]).

Not all the terms of the product of $\hat{\boldsymbol{u}}_{h} \cdot \nabla \boldsymbol{v}_{h}+\nu \Delta \boldsymbol{v}_{h}+\nabla q_{h}$ and the terms that contribute to $\tilde{\boldsymbol{u}}$ in (16) provide stablity. Thus, from the point of view of stability some of them could be neglected. This idea has motivated different term-by-term stabilization methods [18, 19, 12], including the local projection stabilization methods (see for example [20,21, 22]), and motivates also the method proposed next.

\subsection{Term-by-term stabilized formulation}

The method we will propose next is not residual-based, and therefore it is not consistent in the sense used in the finite element context, i.e., the discrete variational problem will not be satisfied when the finite element unknown is replaced by the solution of the continuous problem. However, the consistency error will be of optimal order.

Let us consider expression (17), with $\tilde{P}=P_{h}^{\perp}$. In this case, assuming $P_{h}^{\perp}(\boldsymbol{f}) \approx \mathbf{0}$, we may write:

$$
\frac{\partial \tilde{\boldsymbol{u}}}{\partial t}+\tau_{1}^{-1} \tilde{\boldsymbol{u}}=-P_{h}^{\perp}\left(\hat{\boldsymbol{u}}_{h} \cdot \nabla \boldsymbol{u}_{h}\right)-P_{h}^{\perp}\left(\nabla p_{h}\right)+P_{h}^{\perp}\left(\nu \Delta \boldsymbol{u}_{h}\right) .
$$

All the terms in the RHS have optimal consistency order, in the sense that if we replace the finite element solution by the continuous one and this is smooth enough, the $L^{2}$-norm of these terms behaves as $h^{k+1}$, $k$ being the order of the finite element interpolation. If derivatives of order higher than $k+1$ cannot be considered smooth, the missing powers of $h$ in the interpolation estimates can be compensated by those of the stabilization parameter $\tau_{1}$, whose expression is given below.

Neglecting any of the terms in the RHS of (19) has no consequences in the order of accuracy, but the impact on stability may be very significant. In order to obtain control (stability and convergence) on the convective term and the pressure gradient term, we need to keep the first and the second terms, but we may neglect $P_{h}^{\perp}\left(\nu \Delta \boldsymbol{u}_{h}\right)$. The next idea is to assume that the first two terms are independent. In fact, if $\hat{\boldsymbol{u}}_{h}$ could be replaced by a constant vector field, they would be orthogonal, since the convective term would be solenoidal and the pressure gradient would be potential; there orthogonal components would obviously be also mutually orthogonal.

With the idea described in mind, we may split $\tilde{\boldsymbol{u}}=\tilde{\boldsymbol{u}}_{1}+\tilde{\boldsymbol{u}}_{2}$, where $\tilde{\boldsymbol{u}}_{1}$ and $\tilde{\boldsymbol{u}}_{2}$ are the solution of the evolution problems:

$$
\begin{aligned}
& \frac{\partial \tilde{\boldsymbol{u}}_{1}}{\partial t}+\tau_{1}^{-1} \tilde{\boldsymbol{u}}_{1}=-P_{h}^{\perp}\left(\hat{\boldsymbol{u}}_{h} \cdot \nabla \boldsymbol{u}_{h}\right), \\
& \frac{\partial \tilde{\boldsymbol{u}}_{2}}{\partial t}+\tau_{1}^{-1} \tilde{\boldsymbol{u}}_{2}=-P_{h}^{\perp}\left(\nabla p_{h}\right),
\end{aligned}
$$

both with zero initial condition. Moreover, we may keep only the product of $\tilde{\boldsymbol{u}}_{1}$ with the convective term applied to the test function and the product of $\tilde{\boldsymbol{u}}_{2}$ with the gradient of the pressure test function, since these 
are the terms that provide stability. Altogether, this leads to the stabilized finite element formulation we propose in this work, which consists in finding $\boldsymbol{U}_{h}=\left[\boldsymbol{u}_{h}, p_{h}\right]:(0, T) \rightarrow \boldsymbol{\mathcal { X }}_{h}$ such that

$$
\begin{aligned}
&\left(\frac{\partial \boldsymbol{u}_{h}}{\partial t}, \boldsymbol{v}_{h}\right)+B\left(\boldsymbol{u}_{h} ; \boldsymbol{U}_{h}, \boldsymbol{V}_{h}\right)-\sum_{K}\left(\nabla \cdot \boldsymbol{v}_{h}, \tilde{p}\right)_{K} \\
&-\sum_{K}\left(\boldsymbol{u}_{h} \cdot \nabla \boldsymbol{v}_{h}, \tilde{\boldsymbol{u}}_{1}\right)_{K}-\sum_{K}\left(\nabla q_{h}, \tilde{\boldsymbol{u}}_{2}\right)_{K}=\left\langle\boldsymbol{f}, \boldsymbol{v}_{h}\right\rangle,
\end{aligned}
$$

for all $\left[\boldsymbol{v}_{h}, q_{h}\right] \in \mathcal{X}_{h}$, where the subscales $\tilde{\boldsymbol{u}}_{1}, \tilde{\boldsymbol{u}}_{2}$ are given by (20)-(21) and $\tilde{p}$ by

$$
\tau_{2}^{-1} \tilde{p}=-P_{h}^{\perp}\left(\nabla \cdot \boldsymbol{u}_{h}\right)
$$

The expressions we use for the stabilization parameters $\tau_{1}$ and $\tau_{2}$, appearing in (20)-(23), are

$$
\tau_{1}=\left(\frac{c_{1} \nu}{\left(\frac{h_{1}}{k^{2}}\right)^{2}}+\frac{c_{2}\left|\boldsymbol{u}_{h}\right|}{\frac{h_{2}}{k}}\right)^{-1}, \tau_{2}=\frac{\left(\frac{h_{1}}{k^{2}}\right)^{2}}{c_{1} \tau_{1}},
$$

where $h_{1}$ and $h_{2}$ are characteristic lengths, the first one computed as the square root of the element area in $2 D$ or the cubic root of the element volume in $3 D$, and the second one the element length in the streamline direction. The constants $c_{i}, i=1,2$, are algorithmic parameters in the formulation, while $k$ is the order of the finite element interpolation (see [23]). The values used in this work are $c_{1}=4$ and $c_{2}=2$. These values have proven to be robust in different problems and for different applications. Nevertheless, note that the precise values of the algorithmic constants may affect the error for a given mesh size, but the rate of convergence is unaffected.

The novelty of this method is the tracking of the velocity subscales, two in this case. The quasi-static version of this orthogonal term-by-term method was proposed in [19] and analyzed numerically in [12], and used later in the context of viscoelastic fluids in [15, 24, 14], with better response than the residual version for highly elastic flows. The dynamic version, taking into account the time derivative of the velocity subscales, is required in anisotropic space-time discretizations.

Let us remark that there are other methods for stationary problems that share with ours the introduction of inconsistent terms (not zero when the discrete solution is replaced by the continuous one), but with a consistency error of optimal order. In the finite element context, examples of these methods are the stabilization based on jumps over finite element boundaries (see [25]) or the local projection stabilization (see for example [26]). Apart from the time dependency of the subscales, the latter would give a method similar to (22) replacing the orthogonal projection by the so called "fluctuation operator".

We have presented heretofore a heuristic motivation of the formulation, starting from the VMS framework and using different assumptions. The validity of the resulting formulation will be justified by some analysis and by the numerical performance in the examples presented in Section 5 .

\subsection{About stabilization parameters depending on the time step size}

In $[5,1]$ the importance of the time dependent behavior of the subscales was analyzed in detail. Here we summarize the key ideas for the dynamic term-by-term method proposed in this work.

Using a first order backward difference scheme to discretize in time the subscale equations (20) and (21), 
we obtain:

$$
\begin{aligned}
& \tilde{\boldsymbol{u}}_{1}^{n+1}=\left(\frac{1}{\delta t}+\frac{1}{\tau_{1}}\right)^{-1}\left(\frac{1}{\delta t} \tilde{\boldsymbol{u}}_{1}^{n}-P_{h}^{\perp}\left(\hat{\boldsymbol{u}}_{h}^{n+1} \cdot \nabla \boldsymbol{u}_{h}^{n+1}\right)\right), \\
& \tilde{\boldsymbol{u}}_{2}^{n+1}=\left(\frac{1}{\delta t}+\frac{1}{\tau_{1}}\right)^{-1}\left(\frac{1}{\delta t} \tilde{\boldsymbol{u}}_{2}^{n}-P_{h}^{\perp}\left(\nabla p_{h}^{n+1}\right)\right),
\end{aligned}
$$

where it is understood that $\tau_{1}$ is computed with the velocity at time step $n+1$. From these expressions we see that both velocity subscales depend on $\frac{1}{\tau_{1}^{d}}=\frac{1}{\delta t}+\frac{1}{\tau_{1}}$. Expressions with asymptotic behavior similar to that of $\tau_{1}^{d}$ in terms of $\nu,\left|\hat{\boldsymbol{u}}_{h}\right|, h_{1}, h_{2}$ and $\delta t$ can be found in the specific literature, see for example [9, 27]. In [10] there is a study of the instability encountered when the ASGS method and quasi-static subscales are used. It is shown in that reference that for the Stokes time continuous problem the Schur complement matrix for the pressure is not uniformly invertible, and this property is inherited as $\delta t \rightarrow 0$ if $h$ remains fixed.

It is easily shown that the instability described disappears if

$$
\delta t \geq C \tau_{1}^{n+1}
$$

where $C$ is a positive constant. From this condition it seems clear that the stabilization parameter and the time step size must be related in classical (quasi-static) stabilized finite element methods, since $\tau_{1}$ depends on $h$. It is important to note that, if (27) holds, it is irrelevant from the analysis point of view if the subscales are considered dynamic or quasi-static, since $1 / \delta t$ is at most of the same order as $1 / \tau_{1}$ in (25)-(26). The importance of considering dynamic subscales, reflected in equations (25)-(26) by the presence of $\frac{1}{\delta t} \tilde{\boldsymbol{u}}_{1}^{n}$ and $\frac{1}{\delta t} \tilde{\boldsymbol{u}}_{2}^{n}$, is that (27) is not needed for stability, as it will be shown below. Moreover, we will see in the numerical examples that accuracy is improved when dynamic subscales are considered.

\section{Dissipative structure and stability}

\subsection{Local energy conservation}

Following $[28,6]$ we can examine the conservation structure of the proposed finite element formulation. Defining an arbitrary subdomain $\omega \subset \Omega$ formed by an arbitrary patch of elements and introducing the discrete counterpart of the fluxes $\boldsymbol{h}^{\omega}: \partial \omega \rightarrow \mathbb{R}^{d}$, denoted by $\boldsymbol{h}_{h}^{\omega}$, we can write:

$$
\begin{gathered}
\left(\frac{\partial \boldsymbol{u}_{h}}{\partial t}, \boldsymbol{v}_{h}\right)_{\omega}+\nu\left(\nabla \boldsymbol{u}_{h}, \nabla \boldsymbol{v}_{h}\right)_{\omega}+\left\langle\boldsymbol{u}_{h} \cdot \nabla \boldsymbol{u}_{h}, \boldsymbol{v}_{h}\right\rangle_{\omega}-\left(p_{h}, \nabla \cdot \boldsymbol{v}_{h}\right)_{\omega}+\left(\nabla \cdot \boldsymbol{u}_{h}, q_{h}\right)_{\omega} \\
-\sum_{K \subset \omega}\left(P_{h}^{\perp}\left(\hat{\boldsymbol{u}}_{h} \cdot \nabla \boldsymbol{v}_{h}\right), \tilde{\boldsymbol{u}}_{1}\right)_{K}-\sum_{K \subset \omega}\left(P_{h}^{\perp}\left(\nabla q_{h}\right), \tilde{\boldsymbol{u}}_{2}\right)_{K}-\sum_{K \subset \omega}\left(P_{h}^{\perp}\left(\nabla \cdot \boldsymbol{v}_{h}\right), \tilde{p}\right)_{K} \\
\quad=\left\langle\boldsymbol{f}, \boldsymbol{v}_{h}\right\rangle_{\omega}+\left\langle\boldsymbol{h}_{h}^{\omega}, \boldsymbol{v}_{h}\right\rangle_{\partial \omega} .
\end{gathered}
$$

Note that we have introduced the orthogonal projection in the terms coming from the subgrid scales. The formulation of the discrete problem (22) is unaffected by this addition due to the fact that $\left(\boldsymbol{v}_{h}, \tilde{\boldsymbol{u}}_{i}\right)=$ $\left(P_{h}^{\perp}\left(\boldsymbol{v}_{h}\right), \tilde{\boldsymbol{u}}_{i}\right), i=1,2$. However, this is not the case in a subdomain $\omega$ of the domain $\Omega$ because the introduction of the projection will change the recovered fluxes $\boldsymbol{h}_{h}^{\omega}$.

The analysis begins by taking the test functions equal to the finite element variables, $\boldsymbol{v}_{h}=\boldsymbol{u}_{h}$ and $q_{h}=p_{h}$ 
in (28), arriving at a local energy conservation statement:

$$
\begin{gathered}
\frac{1}{2} \frac{\mathrm{d}}{\mathrm{d} t}\left\|\boldsymbol{u}_{h}\right\|_{\omega}^{2}+\nu\left\|\nabla \boldsymbol{u}_{h}\right\|_{\omega}^{2}+\left\langle\hat{\boldsymbol{u}}_{h} \cdot \nabla \boldsymbol{u}_{h}, \boldsymbol{u}_{h}\right\rangle_{\omega} \\
-\sum_{K \subset \omega}\left(P_{h}^{\perp}\left(\hat{\boldsymbol{u}}_{h} \cdot \nabla \boldsymbol{u}_{h}\right), \tilde{\boldsymbol{u}}_{1}\right)_{K}-\sum_{K \subset \omega}\left(P_{h}^{\perp}\left(\nabla p_{h}\right), \tilde{\boldsymbol{u}}_{2}\right)_{K}-\sum_{K \subset \omega}\left(P_{h}^{\perp}\left(\nabla \cdot \boldsymbol{u}_{h}\right), \tilde{p}\right)_{K} \\
=\left\langle\boldsymbol{f}, \boldsymbol{u}_{h}\right\rangle_{\omega}+\left\langle\boldsymbol{h}_{h}^{\omega}, \boldsymbol{u}_{h}\right\rangle_{\partial \omega} .
\end{gathered}
$$

A physical interpretation of the different terms in Equation (29) can be made. The first term on the LHS represents the temporal variation of the total kinetic energy of the finite element scales in the volume $\omega$, which occurs because of the viscous dissipation represented by the second term of the LHS and the work done against external forces represented by the first term of the RHS. The second term on the RHS represents part of the work done on the control surface $\partial \omega$. The fluxes $\boldsymbol{h}_{h}^{\omega}$ are the variationally consistent tractions; if the continuous problem is considered, we have

$$
\boldsymbol{h}^{\omega}=-p \hat{\boldsymbol{n}}+\nu \hat{\boldsymbol{n}} \cdot \nabla \boldsymbol{u}
$$

and then

$$
\left\langle\boldsymbol{h}^{\omega}, \boldsymbol{u}\right\rangle_{\partial \omega}=-\int_{\partial \omega} \hat{\boldsymbol{n}} \cdot[\boldsymbol{u} p-\nu \nabla k],
$$

which corresponds to part of the second term in (6). The remaining part corresponds to the flux due to convective effects.

With respect to the convective term, different forms can be considered. In the non-conservative form of the convective term considered so far, we have

$$
\left\langle\hat{\boldsymbol{u}}_{h} \cdot \nabla \boldsymbol{u}_{h}, \boldsymbol{u}_{h}\right\rangle_{\omega}=\frac{1}{2} \int_{\partial \omega} \hat{\boldsymbol{n}} \cdot \hat{\boldsymbol{u}}_{h} \boldsymbol{u}_{h}^{2}-\frac{1}{2} \int_{\omega} \boldsymbol{u}_{h}^{2} \nabla \cdot \hat{\boldsymbol{u}}_{h},
$$

whereas in the conservative form we have

$$
\left\langle\nabla \cdot\left(\hat{\boldsymbol{u}}_{h} \boldsymbol{u}_{h}\right), \boldsymbol{u}_{h}\right\rangle_{\omega}=\frac{1}{2} \int_{\partial \omega} \hat{\boldsymbol{n}} \cdot \hat{\boldsymbol{u}}_{h} \boldsymbol{u}_{h}^{2}+\frac{1}{2} \int_{\omega} \boldsymbol{u}_{h}^{2} \nabla \cdot \hat{\boldsymbol{u}}_{h}
$$

The second term vanishes when $\hat{\boldsymbol{u}}_{h}=\boldsymbol{u}$ (the continuous solution) and is not present in (6). Note that only if the convective term is written in skew-symmetric form this term vanishes when $\hat{\boldsymbol{u}}_{h}=\boldsymbol{u}_{h}$.

Finally, we can identify the terms involving the subgrid scale as the ones responsible for the energy transfer between coarse and fine scales. In order to analyze this transfer, let us consider the energy balance of the subgrid component. Multiplying (20) by $\tilde{\boldsymbol{u}}_{1},(21)$ by $\tilde{\boldsymbol{u}}_{2}$ and (23) by $\tilde{p}$, adding and integrating in the patch $\omega$, we have

$$
\begin{gathered}
\frac{1}{2} \frac{\mathrm{d}}{\mathrm{d} t}\left\|\tilde{\boldsymbol{u}}_{1}\right\|_{\omega}^{2}+\frac{1}{2} \frac{\mathrm{d}}{\mathrm{d} t}\left\|\tilde{\boldsymbol{u}}_{2}\right\|_{\omega}^{2}+\tau_{1}^{-1}\left\|\tilde{\boldsymbol{u}}_{1}\right\|_{\omega}^{2}+\tau_{1}^{-1}\left\|\tilde{\boldsymbol{u}}_{2}\right\|_{\omega}^{2}+\tau_{2}^{-1}\|\tilde{p}\|_{\omega}^{2} \\
+\sum_{K \subset \omega}\left(P_{h}^{\perp}\left(\hat{\boldsymbol{u}}_{h} \cdot \nabla \boldsymbol{u}_{h}\right), \tilde{\boldsymbol{u}}_{1}\right)_{K}+\sum_{K \subset \omega}\left(P_{h}^{\perp}\left(\nabla p_{h}\right), \tilde{\boldsymbol{u}}_{2}\right)_{K}+\sum_{K \subset \omega}\left(P_{h}^{\perp}\left(\nabla \cdot \boldsymbol{u}_{h}\right), \tilde{p}\right)_{K}=0,
\end{gathered}
$$

where we have considered $\tau_{1}$ and $\tau_{2}$ constant in $\omega$ to simplify the discussion. In the above equation, the first and second terms on the LHS represent the temporal variation of the subgrid kinetic energy in the patch $\omega$, which occurs because of the dissipation represented by the third, fourth and fifth terms, and the work done 
against external forces represented by the term of the RHS. Note that adding (29) and (30) we obtain, using the conservative form of the convective term,

$$
\begin{gathered}
\frac{1}{2} \frac{\mathrm{d}}{\mathrm{d} t}\left\|\boldsymbol{u}_{h}\right\|_{\omega}^{2}+\frac{1}{2} \frac{\mathrm{d}}{\mathrm{d} t}\left\|\tilde{\boldsymbol{u}}_{1}\right\|_{\omega}^{2}+\frac{1}{2} \frac{\mathrm{d}}{\mathrm{d} t}\left\|\tilde{\boldsymbol{u}}_{2}\right\|_{\omega}^{2}+\nu\left\|\nabla \boldsymbol{u}_{h}\right\|_{\omega}^{2} \\
+\frac{1}{2} \int_{\partial \omega} \hat{\boldsymbol{n}} \cdot \hat{\boldsymbol{u}}_{h} \boldsymbol{u}_{h}^{2}+\frac{1}{2} \int_{\omega} \boldsymbol{u}_{h}^{2} \nabla \cdot \hat{\boldsymbol{u}}_{h}+\tau_{1}^{-1}\left\|\tilde{\boldsymbol{u}}_{1}\right\|_{\omega}^{2}+\tau_{1}^{-1}\left\|\tilde{\boldsymbol{u}}_{2}\right\|_{\omega}^{2}+\tau_{2}^{-1}\|\tilde{p}\|_{\omega}^{2}=\left\langle\boldsymbol{f}, \boldsymbol{u}_{h}\right\rangle_{\omega}+\left\langle\boldsymbol{h}_{h}^{\omega}, \boldsymbol{u}_{h}\right\rangle_{\partial \omega} .
\end{gathered}
$$

It is seen here that when the mesh is fine enough the dissipation of the total energy depends only on the viscosity. By fine enough we mean that $\frac{\left|\hat{\boldsymbol{u}}_{h}\right| h_{1}}{\nu} \ll 1$, so that the stabilization parameter $\tau_{1} \rightarrow \frac{c_{1} h_{1}^{2}}{\nu}$ and consequently $\tau_{2}$ becomes proportional to $\nu$. This means that the dissipation structure in the case of laminar flows (or when the discretization is fine enough to resolve all the scales of the flow) is correctly predicted.

\subsection{Subscales dissipation}

From equation (29), we can define the numerical dissipation of the finite element kinetic energy as

$$
\varepsilon_{h}=-\sum_{K \subset \omega}\left(P_{h}^{\perp}\left(\hat{\boldsymbol{u}}_{h} \cdot \nabla \boldsymbol{u}_{h}\right), \tilde{\boldsymbol{u}}_{1}\right)_{K}-\sum_{K \subset \omega}\left(P_{h}^{\perp}\left(\nabla p_{h}\right), \tilde{\boldsymbol{u}}_{2}\right)_{K}-\sum_{K \subset \omega}\left(P_{h}^{\perp}\left(\nabla \cdot \boldsymbol{u}_{h}\right), \tilde{p}\right)_{K},
$$

and from (30) the production of subgrid energy as

$$
P_{s}=\sum_{K \subset \omega}\left(P_{h}^{\perp}\left(\hat{\boldsymbol{u}}_{h} \cdot \nabla \boldsymbol{u}_{h}\right), \tilde{\boldsymbol{u}}_{1}\right)_{K}+\sum_{K \subset \omega}\left(P_{h}^{\perp}\left(\nabla p_{h}\right), \tilde{\boldsymbol{u}}_{2}\right)_{K}+\sum_{K \subset \omega}\left(P_{h}^{\perp}\left(\nabla \cdot \boldsymbol{u}_{h}\right), \tilde{p}\right)_{K} .
$$

Note that the formulation ensures that the numerical dissipation is exactly the energy production of the subscales with opposite sign $\left(\varepsilon_{h}=-P_{s}\right)$, a remarkable difference respect to residual-based formulations. This property was also shown to hold with the method presented in [29], although using a more involved projection. As is our case, the use of dynamic subscales turns out to be crucial to obtain the correct energy behavior.

\subsection{Stability analysis}

In this Section, we present a simplified stability analysis of the formulation presented above. We consider the linear Oseen problem, that is, taking a constant advection velocity field $\boldsymbol{a}$. As time integration scheme, we consider only the first order backward difference scheme (for simplicity) and for the spatial discretization we consider isotropic and quasi-uniform meshes, so that we can take $h_{2}=h_{1}=h$, the diameter of the finite element partition. These assumptions are not at all essential, but allow us to simplify the analysis. Note that the stabilization parameters under these simplifications will be constant, given by

$$
\tau_{1}=\left(\frac{c_{1} k^{4} \nu+h c_{2} k|\boldsymbol{a}|}{h^{2}}\right)^{-1}, \quad \tau_{2}=\frac{h^{2}}{k^{4} c_{1} \tau_{1}} .
$$

Under the conditions indicated, the problem to be solved is: given $\boldsymbol{u}_{h}^{n}, \tilde{\boldsymbol{u}}_{1}^{n}$ and $\tilde{\boldsymbol{u}}_{2}^{n}$, find $\boldsymbol{u}_{h}^{n+1}, \tilde{\boldsymbol{u}}_{1}^{n+1}, \tilde{\boldsymbol{u}}_{2}^{n+1}$ and $p_{h}^{n+1}$ such that:

$$
\begin{aligned}
& \left(\frac{\boldsymbol{u}_{h}^{n+1}-\boldsymbol{u}_{h}^{n}}{\delta t}, \boldsymbol{v}_{h}\right)+\nu\left(\nabla \boldsymbol{u}_{h}^{n+1}, \nabla \boldsymbol{v}_{h}\right)+\left\langle\boldsymbol{a} \cdot \nabla \boldsymbol{u}_{h}^{n+1}, \boldsymbol{v}_{h}\right\rangle-\left(p_{h}^{n+1}, \nabla \cdot \boldsymbol{v}_{h}\right) \\
& +\left(\nabla \cdot \boldsymbol{u}_{h}^{n+1}, q_{h}\right)-\sum_{K}\left(P_{h}^{\perp}\left(\boldsymbol{a} \cdot \nabla \boldsymbol{v}_{h}\right), \tilde{\boldsymbol{u}}_{1}^{n+1}\right)_{K}-\sum_{K}\left(P_{h}^{\perp}\left(\nabla q_{h}\right), \tilde{\boldsymbol{u}}_{2}^{n+1}\right)_{K}
\end{aligned}
$$




$$
\begin{aligned}
-\sum_{K}\left(P_{h}^{\perp}\left(\nabla \cdot \boldsymbol{v}_{h}\right), \tilde{p}^{n+1}\right)_{K} & =\left\langle\boldsymbol{f}, \boldsymbol{v}_{h}\right\rangle \\
\frac{\tilde{\boldsymbol{u}}_{1}^{n+1}-\tilde{\boldsymbol{u}}_{1}^{n}}{\delta t}+\tau_{1}^{-1} \tilde{\boldsymbol{u}}_{1}^{n+1} & =-P_{h}^{\perp}\left(\boldsymbol{a} \cdot \nabla \boldsymbol{u}_{h}^{n+1}\right), \\
\frac{\tilde{\boldsymbol{u}}_{2}^{n+1}-\tilde{\boldsymbol{u}}_{2}^{n}}{\delta t}+\tau_{1}^{-1} \tilde{\boldsymbol{u}}_{2}^{n+1} & =-P_{h}^{\perp}\left(\nabla p_{h}^{n+1}\right), \\
\tau_{2}^{-1} \tilde{p}^{n+1} & =-P_{h}^{\perp}\left(\nabla \cdot \boldsymbol{u}_{h}^{n+1}\right),
\end{aligned}
$$

for all velocity test functions $\boldsymbol{v}_{h}$ and pressure test functions $q_{h}$.

The analysis of this problem performed below is based on [5].

Let us introduce some additional used notation needed for the analysis. Given a sequence $F=\left\{f^{n}\right\}$, with $n$ ranging between $n=1, \ldots, N$, the number of time intervals of the partition in time, and $X$ a space of functions with norm $\|\cdot\|_{X}$, we say that $F \in l^{p}(X)$ if $\sum_{n=1}^{N} \delta t\left\|f^{n}\right\|_{X}^{p} \leq C<\infty$, and $F \in l^{\infty}(X)$ if $\max _{n=1, \ldots, N}\left\|f^{n}\right\|_{X} \leq C<\infty$. Hereafter, $C$ denotes a generic positive constant. We also write $\delta f^{n}=$ $f^{n+1}-f^{n}$.

Let us remark the assumptions we will need on the data. We will consider that $\boldsymbol{u}^{0} \in \boldsymbol{L}^{2}(\Omega)$, and therefore $\left\|\boldsymbol{u}_{h}^{0}\right\|$ will be bounded uniformly in $h$. We may take zero as initial conditions for the subscale velocities. Concerning the force term, the classical assumption $\boldsymbol{f} \in L^{2}\left(0, T ; \boldsymbol{H}^{-1}(\Omega)\right)$ could be considered. Since our interest is to prove stability estimates independent of $\nu$ and $\boldsymbol{a}$, a strengthened assumption will be needed, namely, $\boldsymbol{f} \in L^{2}\left(0, T ; \boldsymbol{L}^{2}(\Omega)\right)$. In what follows, we consider $T$ fixed and bounded. For the time discrete problem, the counterpart of $\boldsymbol{f} \in \boldsymbol{L}^{2}\left(0, T ; \boldsymbol{L}^{2}(\Omega)\right)$ is $\left\{\boldsymbol{f}^{n}\right\} \in l^{2}\left(\boldsymbol{L}^{2}(\Omega)\right)$.

Theorem 1 . Let $\boldsymbol{u}_{h}^{n+1}$ and $p_{h}^{n+1}$ be the solution of (32) and $\tilde{\boldsymbol{u}}_{1}^{n+1}, \tilde{\boldsymbol{u}}_{2}^{n+1}$ and $\tilde{p}^{n+1}$ the solutions of (33)-(35), respectively. The following stability bounds hold for all $\delta t>0$ :

$$
\begin{gathered}
\max _{n=1, \ldots, N-1}\left\{\left\|\boldsymbol{u}_{h}^{n+1}\right\|^{2}+\left\|\tilde{\boldsymbol{u}}_{1}^{n+1}\right\|^{2}+\left\|\tilde{\boldsymbol{u}}_{2}^{n+1}\right\|^{2}\right\} \\
+\sum_{n=1}^{N-1} \delta t\left(\nu\left\|\nabla \boldsymbol{u}_{h}^{n+1}\right\|^{2}+\left\|\tau_{1}^{-\frac{1}{2}} \tilde{\boldsymbol{u}}_{1}^{n+1}\right\|^{2}+\left\|\tau_{1}^{-\frac{1}{2}} \tilde{\boldsymbol{u}}_{2}^{n+1}\right\|^{2}\right) \leq C\left(\sum_{n=1}^{N-1} \delta t\left\|\boldsymbol{f}^{n+1}\right\|^{2}+\left\|\boldsymbol{u}^{0}\right\|^{2}\right) .
\end{gathered}
$$

Therefore, if $\left\{\boldsymbol{f}^{n}\right\} \in l^{2}\left(\boldsymbol{L}^{2}(\Omega)\right)$ and $\boldsymbol{u}^{0} \in \boldsymbol{L}^{2}(\Omega)$, we have that

$$
\begin{gathered}
\left\{\boldsymbol{u}_{h}^{n}\right\} \in l^{\infty}\left(\boldsymbol{L}^{2}(\Omega)\right),\left\{\tilde{\boldsymbol{u}}_{1}^{n}\right\} \in l^{\infty}\left(\boldsymbol{L}^{2}(\Omega)\right), \quad\left\{\tilde{\boldsymbol{u}}_{2}^{n}\right\} \in l^{\infty}\left(\boldsymbol{L}^{2}(\Omega)\right), \\
\nu^{\frac{1}{2}}\left\{\boldsymbol{u}_{h}^{n}\right\} \in l^{2}\left(\boldsymbol{H}^{1}(\Omega)\right),\left\{\tau_{1}^{-\frac{1}{2}} \tilde{\boldsymbol{u}}_{1}^{n}\right\} \in l^{2}\left(\boldsymbol{L}^{2}(\Omega)\right),\left\{\tau_{1}^{-\frac{1}{2}} \tilde{\boldsymbol{u}}_{2}^{n}\right\} \in l^{2}\left(\boldsymbol{L}^{2}(\Omega)\right) .
\end{gathered}
$$

Proof. In order to obtain stability bounds for the finite element solution, we test (32) by $\boldsymbol{v}_{h}=\boldsymbol{u}_{h}^{n+1}$ and $q_{h}=p_{h}^{n+1}$. Since $\boldsymbol{a}$ is constant and $\boldsymbol{u}_{h}^{n+1}=\mathbf{0}$ on $\partial \Omega,\left\langle\boldsymbol{a} \cdot \nabla \boldsymbol{u}_{h}^{n+1}, \boldsymbol{u}_{h}^{n+1}\right\rangle=0$. On the other hand, $\left\langle\boldsymbol{f}^{n+1}, \boldsymbol{u}_{h}^{n+1}\right\rangle \leq \frac{\alpha}{2}\left\|\boldsymbol{f}^{n+1}\right\|^{2}+\frac{1}{2 \alpha}\left\|\boldsymbol{u}_{h}^{n+1}\right\|^{2}$ for all $\alpha>0$. Adding up the resulting inequalities from $n=0$ to an arbitrary time level $M$, and using the discrete Gronwall Lemma with $\alpha>1$, we get

$$
\begin{array}{r}
\frac{1}{2}\left\|\boldsymbol{u}_{h}^{M+1}\right\|^{2}+\frac{1}{2} \sum_{n=0}^{M}\left\|\delta \boldsymbol{u}_{h}^{n}\right\|^{2}+\sum_{n=0}^{M} \delta t \nu\left\|\nabla \boldsymbol{u}_{h}^{n+1}\right\|^{2} \\
-\sum_{n=0}^{M} \delta t\left(P_{h}^{\perp}\left(\boldsymbol{a} \cdot \nabla \boldsymbol{u}_{h}^{n+1}\right), \tilde{\boldsymbol{u}}_{1}^{n+1}\right)_{K}-\sum_{n=0}^{M} \delta t\left(P_{h}^{\perp}\left(\nabla p_{h}^{n+1}\right), \tilde{\boldsymbol{u}}_{2}^{n+1}\right)_{K}
\end{array}
$$




$$
-\sum_{n=0}^{M} \delta t\left(P_{h}^{\perp}\left(\nabla \cdot \boldsymbol{u}_{h}^{n+1}\right), \tilde{p}^{n+1}\right)_{K}=C\left(\sum_{n=0}^{M} \delta t\left\|\boldsymbol{f}^{n+1}\right\|^{2}+\left\|P_{h}\left(\boldsymbol{u}^{0}\right)\right\|^{2}\right) .
$$

Now we can multiply (33) by $\tilde{\boldsymbol{u}}_{1}^{n+1}$, integrate over the whole domain and add up the result from $n=0$ to $n=M$. Doing that we obtain:

$$
\frac{1}{2}\left\|\tilde{\boldsymbol{u}}_{1}^{M+1}\right\|^{2}+\frac{1}{2} \sum_{n=0}^{M}\left\|\delta \tilde{\boldsymbol{u}}_{1}^{n}\right\|^{2}+\sum_{n=0}^{M} \delta t\left\|\tau_{1}^{-\frac{1}{2}} \tilde{\boldsymbol{u}}_{1}^{n+1}\right\|^{2}=-\sum_{n=0}^{M} \delta t\left(P_{h}^{\perp}\left(\boldsymbol{a} \cdot \nabla \boldsymbol{u}_{h}^{n+1}\right), \tilde{\boldsymbol{u}}_{1}^{n+1}\right) .
$$

In similar way, we can multiply (34) by $\tilde{\boldsymbol{u}}_{2}^{n+1}$ and (35) by $\tilde{p}^{n+1}$ and integrate over the whole domain and add up the result from $n=0$ to $n=M$, obtaining:

$$
\begin{aligned}
\frac{1}{2}\left\|\tilde{\boldsymbol{u}}_{2}^{M+1}\right\|^{2}+\frac{1}{2} \sum_{n=0}^{M}\left\|\delta \tilde{\boldsymbol{u}}_{2}^{n}\right\|^{2}+\sum_{n=0}^{M} \delta t\left\|\tau_{1}^{-\frac{1}{2}} \tilde{\boldsymbol{u}}_{2}^{n+1}\right\|^{2} & =-\sum_{n=0}^{M} \delta t\left(P_{h}^{\perp}\left(\nabla p_{h}^{n+1}\right), \tilde{\boldsymbol{u}}_{2}^{n+1}\right) \\
\sum_{n=0}^{M} \delta t\left\|\tau_{2}^{-\frac{1}{2}} \tilde{p}^{n+1}\right\|^{2} & =-\sum_{n=0}^{M} \delta t\left(P_{h}^{\perp}\left(\nabla \cdot \boldsymbol{u}_{h}^{n+1}\right), \tilde{p}^{n+1}\right) .
\end{aligned}
$$

Adding (37)-(40), we arrive to

$$
\begin{array}{r}
\left\|\boldsymbol{u}_{h}^{M+1}\right\|^{2}+\left\|\tilde{\boldsymbol{u}}_{1}^{M+1}\right\|^{2}+\left\|\tilde{\boldsymbol{u}}_{2}^{M+1}\right\|^{2}+\sum_{n=0}^{M}\left\|\delta \boldsymbol{u}_{h}^{n}\right\|^{2}+\sum_{n=0}^{M}\left\|\delta \tilde{\boldsymbol{u}}_{1}^{n}\right\|^{2}+\sum_{n=0}^{M}\left\|\delta \tilde{\boldsymbol{u}}_{2}^{n}\right\|^{2} \\
+\sum_{n=0}^{M} \delta t \nu\left\|\nabla \boldsymbol{u}_{h}^{n+1}\right\|^{2}+\sum_{n=0}^{M} \delta t\left\|\tau_{1}^{-\frac{1}{2}} \tilde{\boldsymbol{u}}_{1}^{n+1}\right\|^{2}+\sum_{n=0}^{M} \delta t\left\|\tau_{1}^{-\frac{1}{2}} \tilde{\boldsymbol{u}}_{2}^{n+1}\right\|^{2}+\sum_{n=0}^{M} \delta t\left\|\tau_{2}^{-\frac{1}{2}} \tilde{p}^{n+1}\right\|^{2} \\
\leq C\left(\sum_{n=0}^{M} \delta t\left\|\boldsymbol{f}^{n+1}\right\|^{2}+\left\|P_{h}\left(\boldsymbol{u}^{0}\right)\right\|^{2}\right)
\end{array}
$$

from where the theorem follows.

The results proved in Theorem 1 give stability for both the finite element component and the subscales. However, we are interested only in the stability of the finite element solution. The question that arises is therefore what does the stability for the subscales mean in terms of the finite element solution. Following the same steps as in [5], we could show that the control obtained for $\tilde{\boldsymbol{u}}_{1}$ can be translated into control on the convective term and the control on $\tilde{\boldsymbol{u}}_{2}$ implies control on the pressure gradient.

\section{Numerical Results}

In this Section, some test are conducted to show the numerical performance of the proposed stabilized formulation in order to illustrate the importance of a VMS method with dynamic subscales. The first example (Sub-Section 5.1) is the classical flow over a cylinder problem at Reynolds number Re $=100$, where the formulation is applied with a small time step in order to show numerically the result proved in Theorem 1. The second example (Sub-Section 5.2) corresponds to a classical thermally coupled flow benchmark, the $8: 1$ differentially heated cavity, used to prove the capability of the proposed formulation to solve highly convective cases (even chaotic). Finally, in Sub-Section 5.3 a three-dimensional problem is solved to show that the formulation works in three dimensional cases. 


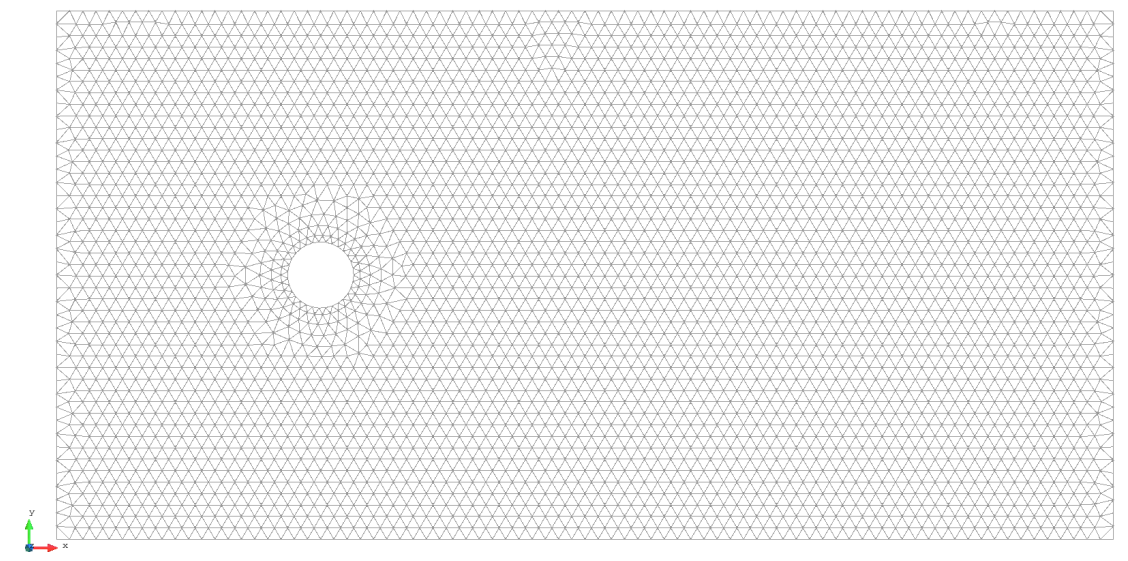

Figure 1: Mesh used for the flow over a cylinder problem.

The time integration scheme used in all numerical cases is the second order backward differencing scheme (Equation (10)) for the finite element part and the first order backward differencing scheme (Equation (9)) for the time integration of the subscales.

\subsection{Flow Over a Cylinder at Reynolds number $\mathrm{Re}=100$}

The flow over a cylinder is a classical benchmark to test new formulations. In this Sub-Section we use it to prove that only with VMS methods with dynamic subscales space-time anisotropic discretizations can be used without any restriction.

The mesh used to solve this first numerical example is shown in Figure 1; as it can be seen, it is a very coarse mesh, and was only selected to prove that the proposed method does not need to satisfy condition (27). The computational domain is $\bar{\Omega}=[0,16] \times[-4,4] \backslash D$, with the cylinder $D$ of unitary diameter and centered at point $(4,0)$. No sensitivity analysis was done to prove the independence of the domain size to ensure that the solution represents a non-confined problem, which is not the purpose of this work. The same unstructured mesh was used with linear and quadratic triangular elements. In the case of linear elements the mesh consists of 2098 nodes and 3762 elements, while in the quadratic case the mesh consists of 7958 nodes. In both cases the minimum element size was imposed over the cylinder, with a value equal to 0.1, while on the other regions the element size was set to 0.2 . As boundary conditions, the inflow velocity at $x=0$ is prescribed to $(1,0)$, whereas at $y=-4$ and $y=4$, the $y$-velocity component is prescribed to 0 and the $x$-velocity is left free. For the outflow condition $(x=16)$ both velocity components are left free. To ensure that the Reynolds number (based on the inflow velocity and the cylinder diameter) is $R e=100$, the kinematic viscosity is set to $\nu=0.01$.

To prove numerically that the stability of the dynamic term-by-term stabilized formulation proposed is independent of the space-time discretization, we solve the problem for the coarse mesh shown in Figure 1 up to $\delta t \approx \tau_{1, \min }$ for linear and quadratic elements, where $\tau_{1 \text {,min }}$ is the minimum value of $\tau_{1}$ computed over the whole domain (note that it needs to be evaluated at the numerical integration points within each element). As shown in [30], $\tau_{1, \text { min }}$ behaves as the critical time step of the explicit Euler time integration scheme for the convective and viscous terms (pressure needs to be treated implicitly to impose incompressibility). We 
will show that the quasi-static version of the formulation begins to be unstable when $\delta t$ is approximately in the range $22 \tau_{1, \min } \leq \delta t \leq 44 \tau_{1, \text { min }}$ for linear elements and in the range $4 \tau_{1, \text { min }} \leq \delta t \leq 9 \tau_{1, \text { min }}$ for quadratic elements, and no solution can be obtained for smaller time step sizes. With the aim to compare the term-byterm formulation proposed in this work and a residual-based one, we also solve the same problem using the orthogonal residual-based VMS formulation proposed in [4]. This allows us to evaluate how dissipative the present formulation is with respect to the residual-based one. In Table 1, we refer to the dynamic version of the term-by-term stabilization method as D-SOSGS, its quasi-static version as SOSGS, while the residualbased orthogonal VMS method is referred to as D-OSGS and OSGS for its dynamic and quasi-static versions, respectively.

\begin{tabular}{|c|c|c|c|c|c|}
\hline$P_{1}$ & $\delta t=0.1$ & $\delta t=0.05$ & $\delta t=0.025$ & $\delta t=0.0125$ & $\delta t=1.5625 \times 10^{-3}$ \\
$\tau_{1, \text { min }} \approx 1.135 \times 10^{-3}$ & & & & - \\
OSGS & Solved & Unsolved & Unsolved & Solved & Solved \\
D-OSGS & Solved & Solved & Solved & - \\
SOSGS & Solved & Solved & Unsolved & - & Solved \\
D-SOSGS & Solved & Solved & Solved & Solved \\
\hline$P_{2}$ & $\delta t=0.1$ & $\delta t=0.00625$ & $\delta t=0.003125$ & $\delta t=1.5625 \times 10^{-3}$ & $\delta t=9.7656 \times 10^{-5}$ \\
$\tau_{1, \text { min }} \approx 7.3 \times 10^{-5}$ & & & & - & - \\
OSGS & Solved & Solved & Unsolved & Solved & Solved \\
D-OSGS & Solved & Solved & Solved & Unsolved & - \\
SOSGS & Solved & Solved & Solved & Solved & Solved \\
D-SOSGS & Solved & Solved & Solved & &
\end{tabular}

Table 1: Summary of solved and unsolved cases in flow over a cylinder problem.

The pressure contour lines and the streamlines obtained with the dynamic term-by-term formulation are showed in Figure 2 for the mesh showed in Figure 1 and for $\delta t=0.1$, for linear (top) and quadratic (bottom) elements. Note that the differences in the results between linear and quadratic elements come from the much better discretization of the latter case.

The beginning of the instability caused by the space-time discretization restriction can be identified as an irregular evolution of some field, the pressure in the case considered, as we can see in Figure 3 (top right figure). There, the vertical velocity and the pressure evolution are plotted at a point located downstream the cylinder in the mid plane and for the smallest time step size that can be used for all methods using linear elements. In this figure, we can see that the quasi-static term-by-term formulation is the most diffusive, followed by the quasi-static version of the orthogonal residual-based one, and the less dissipative method is the dynamic residual-based method. As quantitative comparison, the periods of the oscillations obtained using the different formulation are: 6.85 for the OSGS formulation, 6.35 for the DOSGS formulation, 7.25 for the SOSGS method and 6.6 for the DSOSGS method, in all cases for the coarse mesh showed in Figure 1 and for $\delta t=0.1$. Note that as reference value for the period we can use 6.035 , proposed in [31], where this problem was analyzed in detail. In the bottom plots of Figure 3 , the same point is tracked for the case $\delta t \approx \tau_{1, \min }$ for both dynamic formulations, the term-by-term and the residual-based one. For this case with a time step that can be considered very small, we can see as both formulations can solve the problem, corroborating the stability results proved in Section 4. It is remarkable that the term-by-term formulation in this case yields a smoother solution than the one obtained with the residual-based formulation; this may be due to the more dissipative response of the term-by-term formulation observed in this first numerical test. The differences in 

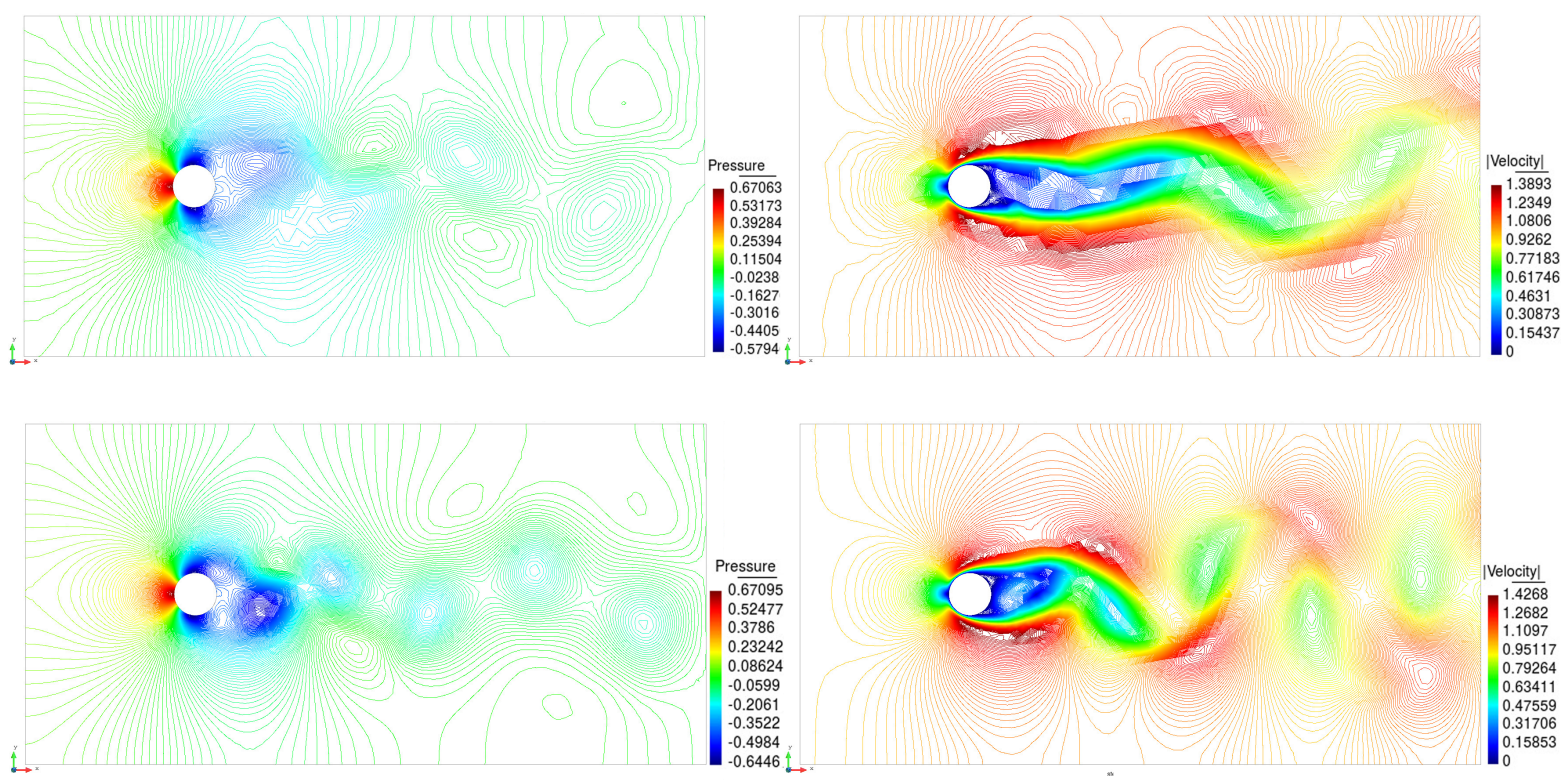

Figure 2: Pressure contourlines and streamlines for the flow over a cylinder problem for linear elements (top) and quadratic elements (bottom).
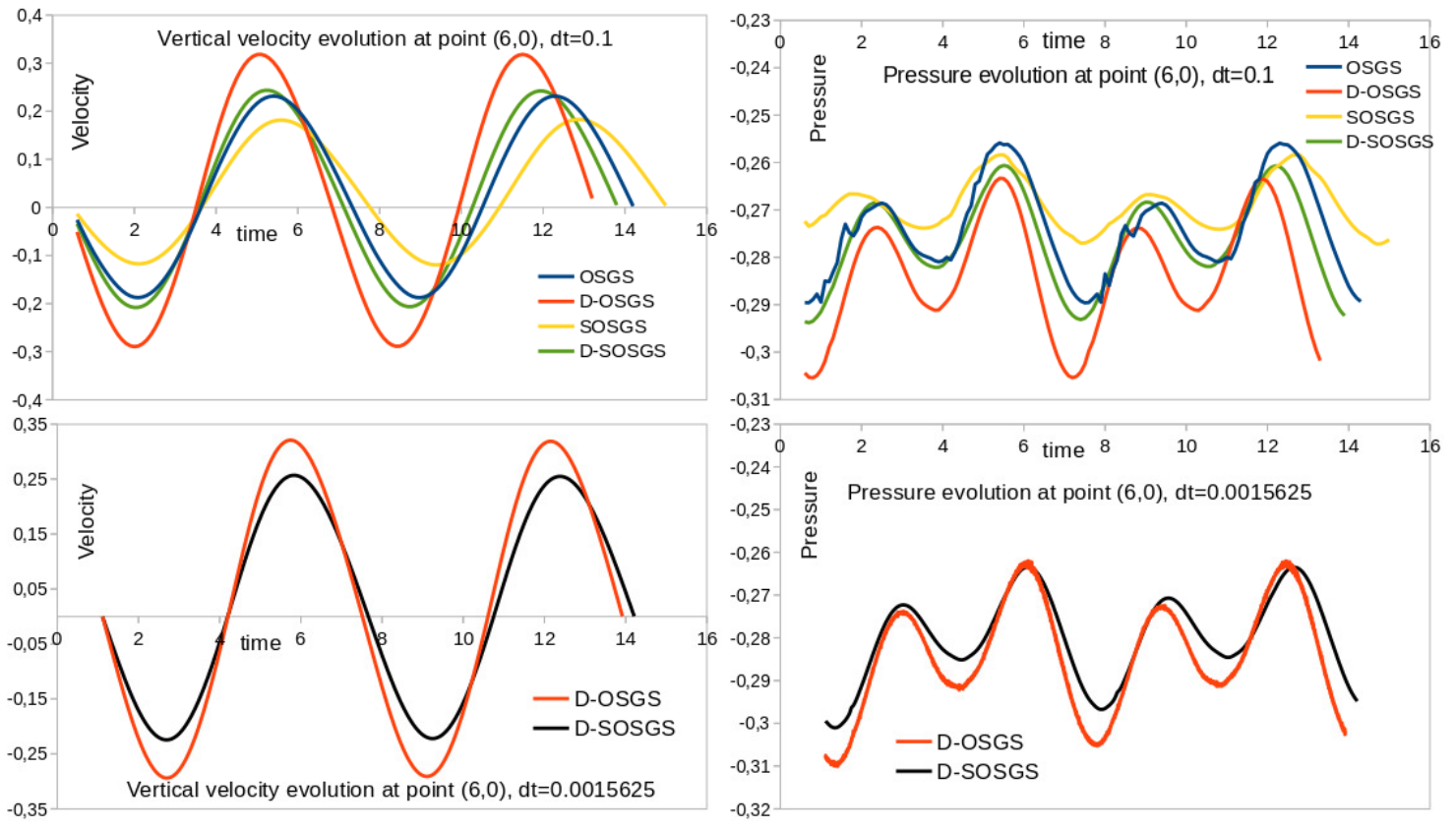

Figure 3: Vertical velocity and pressure evolution at point $(6,0)$ for linear elements and for different time step sizes. 

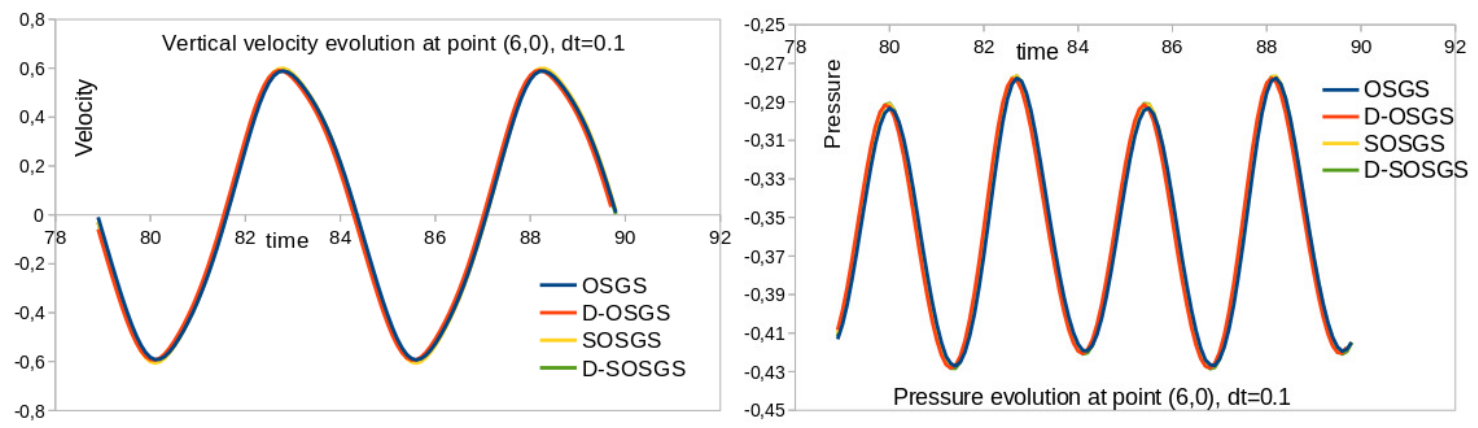

Figure 4: Vertical velocity and pressure evolution in point $(6,0)$ for quadratic elements and for $\delta t=0.05$.

the pressure and velocity values between the residual-based and the term-by-term formulations appreciated in Figure 3 are due to the coarse spatial discretization used; for finer meshes, the results overlap.

The same point was tracked for quadratic elements. The vertical velocity and pressure evolution are plotted in Figure 4 using $\delta t=0.05$. In this case, all methods consider yield very similar results.

An important result is reported in Figure 5. The design of the term-by-term stabilized formulation that we have proposed from the VMS framework relies on the fact that the vector fields $-P_{h}^{\perp}\left(\hat{\boldsymbol{u}}_{h} \cdot \nabla \boldsymbol{u}_{h}\right)$ and $-P_{h}^{\perp}\left(\nabla p_{h}\right)$ in Equation (19) are independent. In particular, we have argued that this would be true if the former were a component of a solenoidal field and the latter a component of a potential field. This independence has permitted the introduction of the subscales $\tilde{\boldsymbol{u}}_{1}$ and $\tilde{\boldsymbol{u}}_{2}$, so that $\tilde{\boldsymbol{u}}=\tilde{\boldsymbol{u}}_{1}+\tilde{\boldsymbol{u}}_{2}$. Now we may verify a posteriori whether this assumption is (approximately) true or not. To this end, in Figure 5 the subscale velocity components are plotted for the same point as for Figures 3 and 4 . The nomenclature used in Figure 5 is as follows: Usgs and Vsgs are the velocity subscale components obtained using the orthogonal residual-based VMS method, $\mathrm{Usgs}_{i}$ and $\operatorname{Vsgs}_{i}$ with $i=1$ and 2, are the subscale velocity components obtained using the term-by-term method (when $i=1$ we refer to the convective subscale velocity and when $i=2$ we refer to the pressure gradient subscale velocity) and, finally, Usgs 12 and $\mathrm{Vsgs}_{12}$ represent the sum of both subscale velocities for each component.

For linear elements, the difference between the subscale velocities obtained using the residual-based formulation and those obtained using the term-by-term formulation come from the cross local inner terms, and therefore, the subscale velocities do not necessarily have to be equal in both methods. However, as we can see in Figure 5 (top), the sum of both velocity subscales is very close to the subscale obtained using the residual-based formulation, and the shape of the curve is exactly the same. The results for quadratic elements are shown in Figure 5 (bottom). In this case, the presence of the Laplacian term in the residual-based formulation increases the difference between the subscale velocities of the two methods compared; however, again the shape of the curves is the same.

\subsection{Flow in a differentially heated cavity with aspect ratio 8}

In thermally coupled problems, the $8: 1$ aspect ratio planar cavity is a common benchmark to check new formulations. In this work, we use it to show that the dynamic term-by-term formulation is highly robust even in chaotic cases.

The thermal model we consider is based on the simplest Bossinesq assumption. In this case, the body force $\boldsymbol{f}$ in the momentum equation (1) is given by $\boldsymbol{f}=\beta \boldsymbol{g}\left(T-T_{\mathrm{ref}}\right)$, where $\beta$ is the thermal expansion 

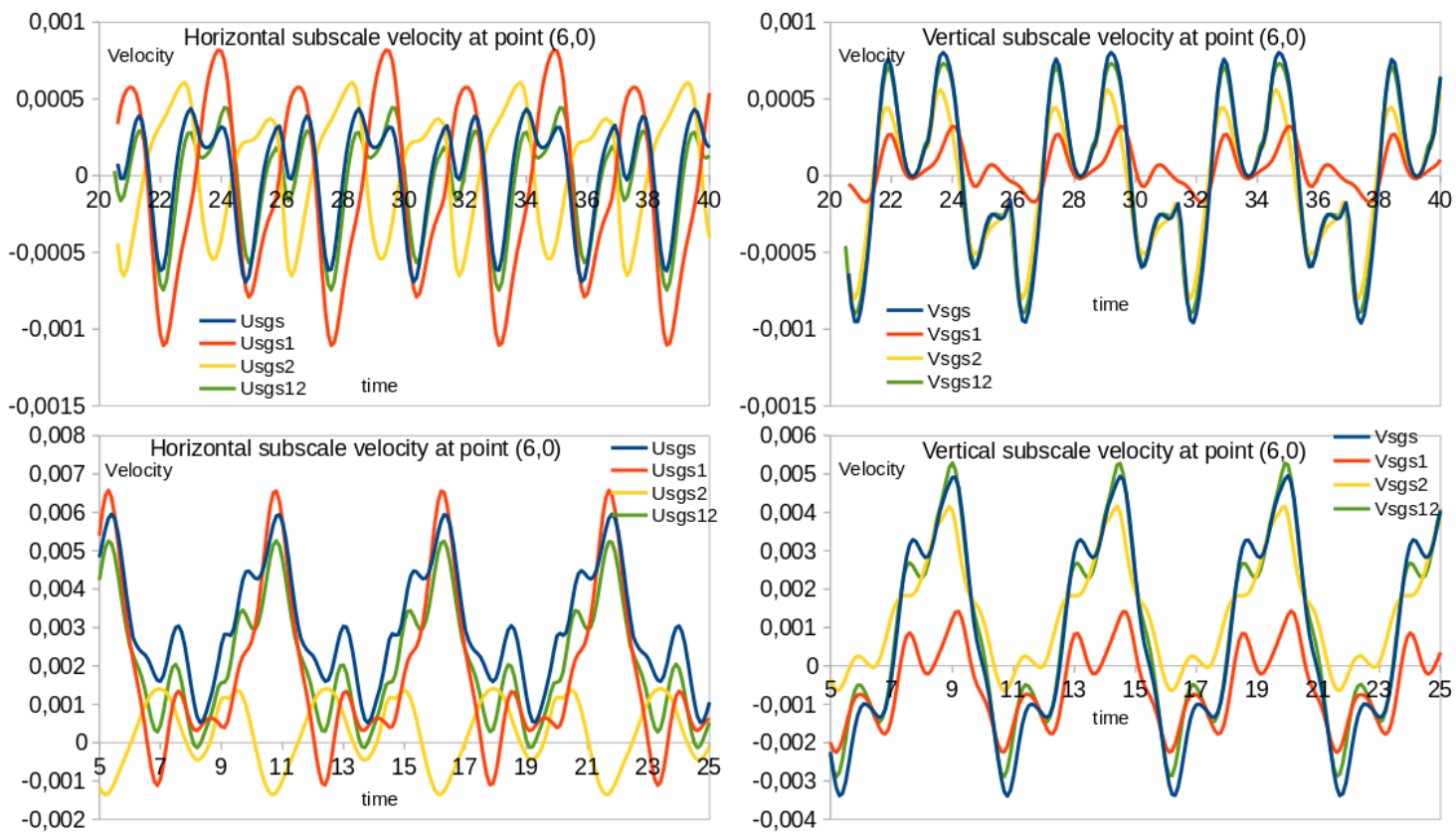

Figure 5: Subscale velocity components evolution for linear elements (top) and quadratic elements (bottom).

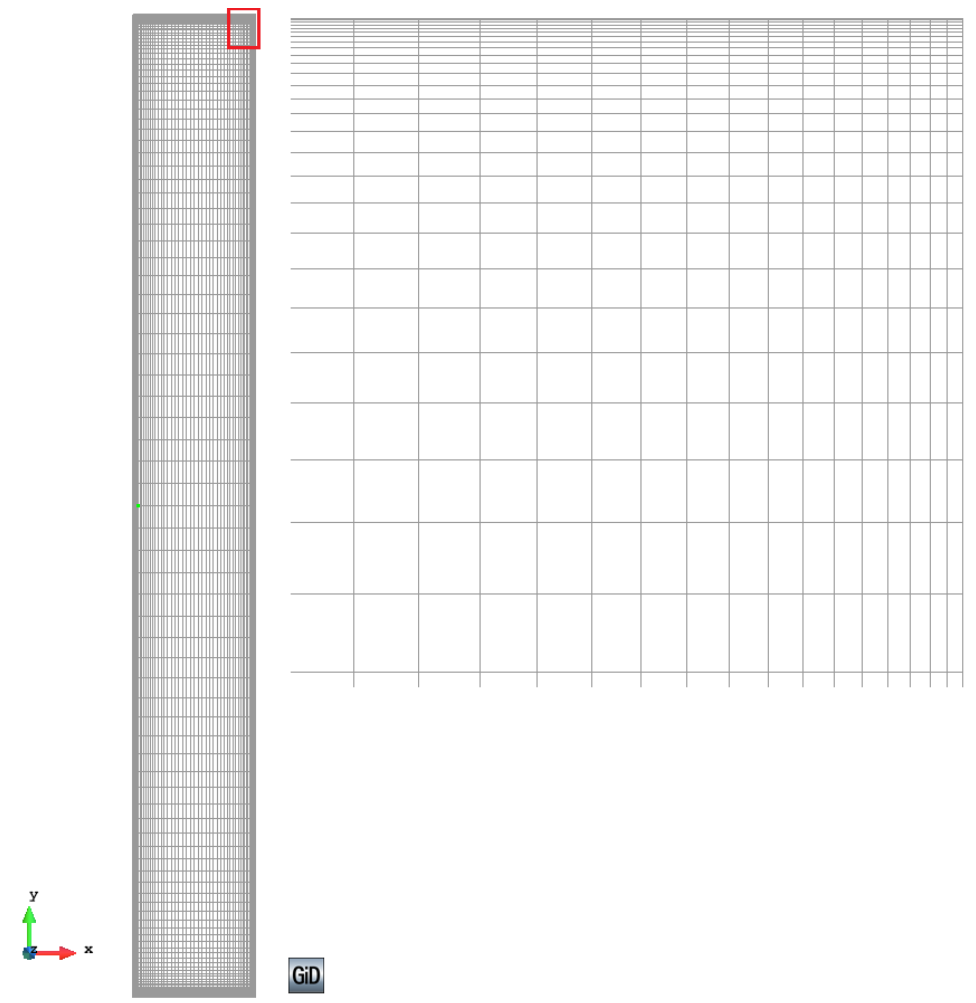

Figure 6: Mesh used for the $8: 1$ planar differentially heated cavity problem. Full domain (right), and corner zoomed by 25 (right). 
coefficient, $\boldsymbol{g}$ the gravity acceleration vector (of norm $g$ ), $T$ the temperature (not time, now) and $T_{\text {ref }}$ the reference temperature. The temperature is obtained by solving the energy equation. The Navier-Stokes equations are solved using the formulation presented in this work, whereas the heat equation is solved using the orthogonal subgrid scale approach detailed in [32].

The geometry of the problem is defined as a rectangle eight times higher than wide. The computational domain is defined as $[0,1] \times[0,8]$. The mesh used is a structured one, exponentially concentrated close to the walls, formed by 6000 linear quatrilateral elements and 6171 nodal points; it is showed in Figure 6 . As velocity boundary conditions, the non-slip prescription is imposed on all walls, while as temperature conditions the left wall is defined as the hot wall, with a temperature $T_{H}=600$, while the left wall is defined as a cold wall, with a temperature $T_{C}=300$. The horizontal walls are set as adiabatic.

The Rayleigh number defined as $\mathrm{Ra}=\frac{\rho^{2} g \beta c_{p}\left(T_{H}-T_{C}\right) L^{3}}{\kappa \mu}$ is the dimensionless number that defines the type and the regime of the flow. In this definition, $c_{p}$ the specific heat, $\kappa$ the conductivity of the fluid and $L$ a characteristic length, taken in our case as the cavity width.

In Figures 7 and 8 some qualitative results for this problem are presented. In Figure 7 the temperature contour-lines and the streamlines are presented for a periodic case (first bifurcation) defined by $\mathrm{Ra}=3.45 \times 10^{5}$ and for a chaotic case, in which $\mathrm{Ra}=1 \times 10^{7}$. The time step size used to solve these cases was $\delta t=0.1$ for the periodic case and $\delta t=0.025$ for the chaotic one.

The subscale variables are calculated at the numerical integrations points within each element domain. In Figure 8, the magnitude of the finite element velocity and the magnitude of the subscale velocities are presented as contours at the nodal points. In the legend, Vesgs 1 represents the convective subscale velocity magnitude, while Vesgs 2 represents the pressure gradient subscale velocity magnitude, in both cases using an automatic smoothing (projection) from the integration points to the nodes (this is why some negative values are obtained).

It is well known for the $8: 1$ planar case of the differentially heated cavity that the critical Rayleigh number (first Hopf bifurcation) that gives place to a time dependent flow is close to $3.45 \times 10^{5}$. For this case, both the quasi-static and dynamic term-by-term formulations work well. However, for Ra $=1 \times 10^{7}$, where the flow is chaotic, the quasi-static version begins to show signs of instability, if the time step size is reduced. For chaotic flows it is crucial for a good resolution of the physics of the problem to be able to use very small time step sizes. In Figure 9, the phase diagram for the quasi-static (left) and for the dynamic (right) versions of the term-by-term stabilized finite element formulation are presented using a time step size of $\delta t=0.003125$. Note that for this case $\tau_{1, \min } \approx 0.5 \times 10^{-4}$. It is evident than the dynamic version yields a smother curve. A similar pattern has been reported for the residual-based VMS formulation in [33].

\subsection{Cubic Lid-driven cavity flow}

The cubic lid-driven cavity flow problem is a classical benchmark used in the three-dimensional case. According to the numerical results presented in [34], who investigated a set of subcritical and slightly supercritical flows in a cubic lid-driven cavity, the steady transition occurs via a subcritical Hopf bifurcation at a Reynolds number $\operatorname{Re}_{\mathrm{cr}} \approx 1914$, being this Reynolds number based on the length of the side of the cube and the velocity prescribed on the top lid (zero velocity is prescribed elsewhere). In a more recent experimental work [35], the steady-unsteady transition has been defined over the range $1700<\operatorname{Re}_{\mathrm{cr}}<1970$. Beyond a Reynolds number Re $>1970$, the flow becomes oscillatory. 


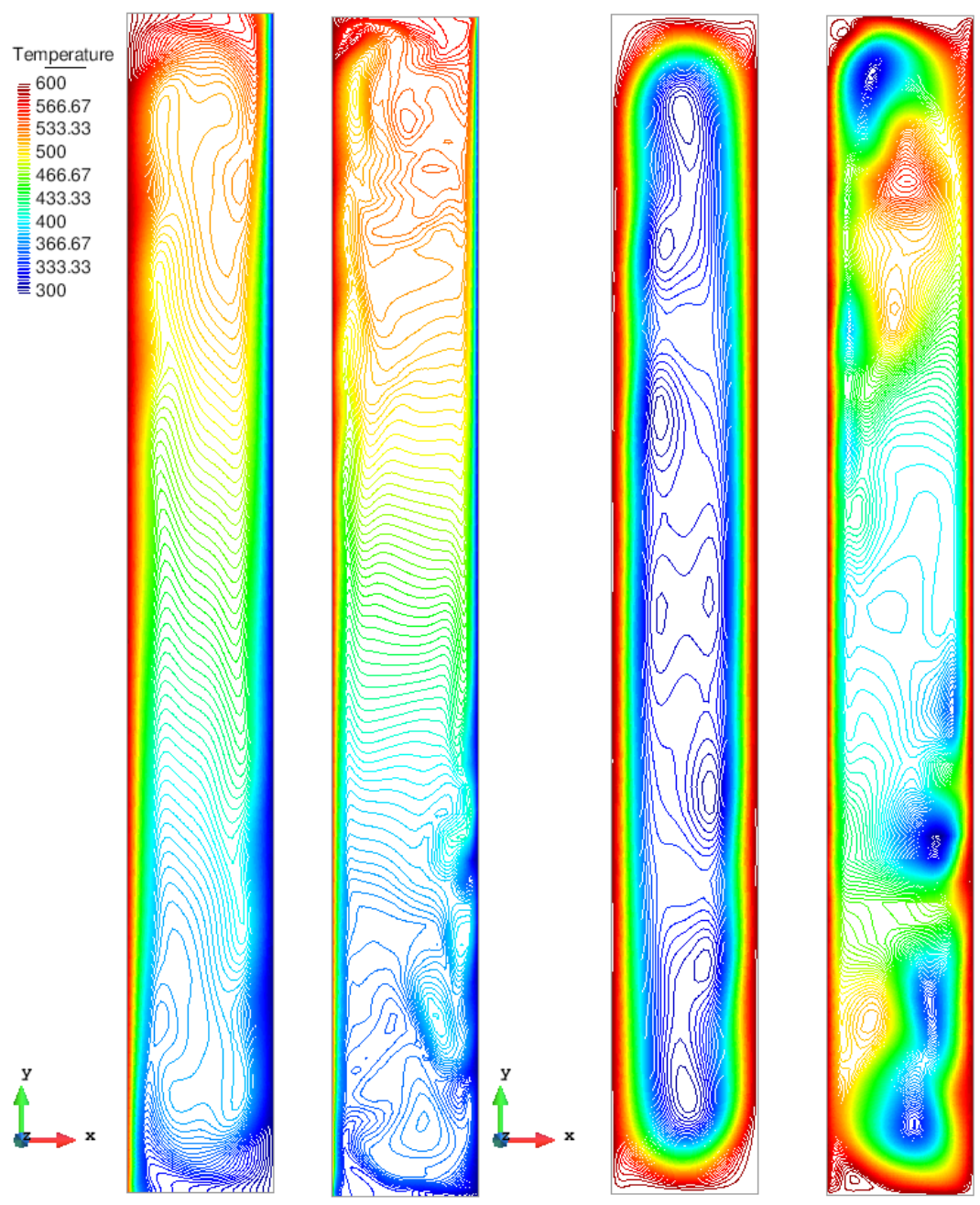

Figure 7: Temperature countourlines (left couple) and streamlines (right couple) for the $8: 1$ planar cavity, for Ra $=3.45 \times 10^{5}$ (left in each couple) and $\mathrm{Ra}=1 \times 10^{7}$ (right in each couple). 

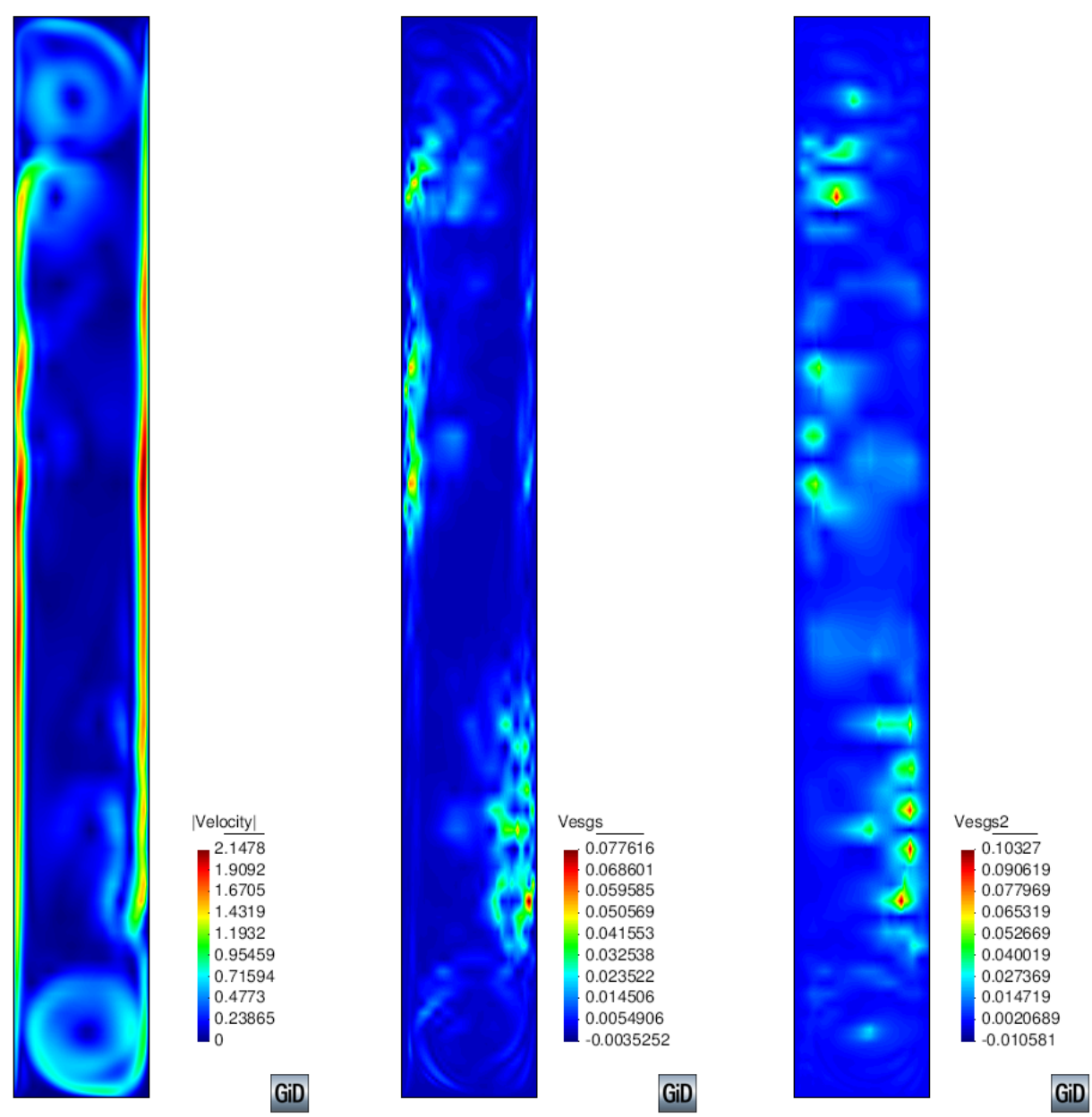

Figure 8: Magnitude of velocity, from left to right: finite element velocity, convective velocity subscale and pressure gradient velocity subscale for the $\mathrm{Ra}=1 \times 10^{7}$ case.
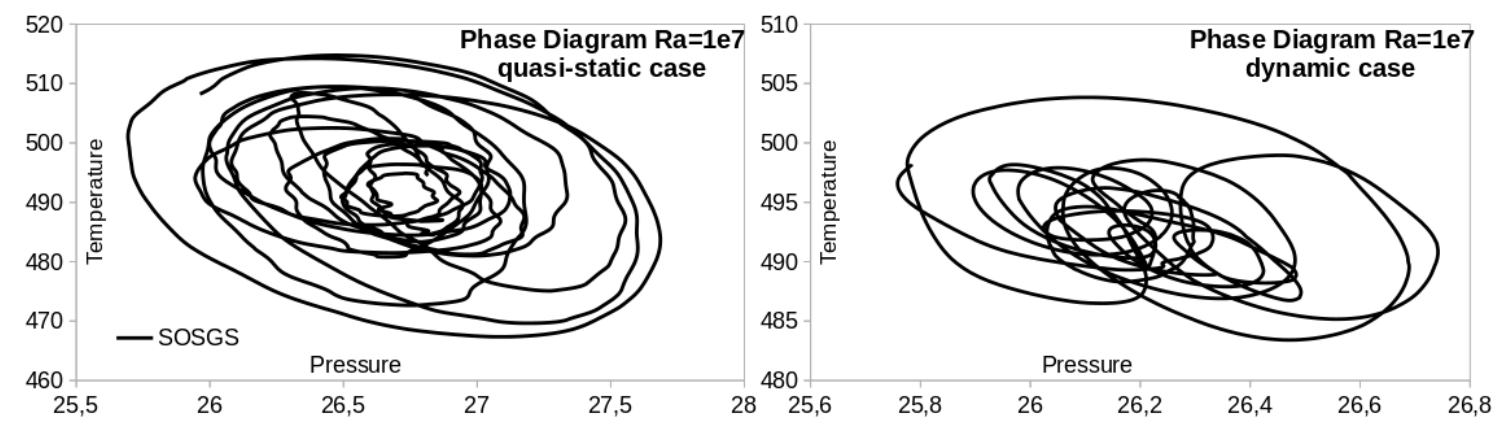

Figure 9: Phase diagram (pressure v/s temperature) for $\mathrm{Ra}=1 \times 10^{7}$ at point $(0.05,4)$ : quasi-static term-by-term formulation (left) and dynamic term-by-term (right). 

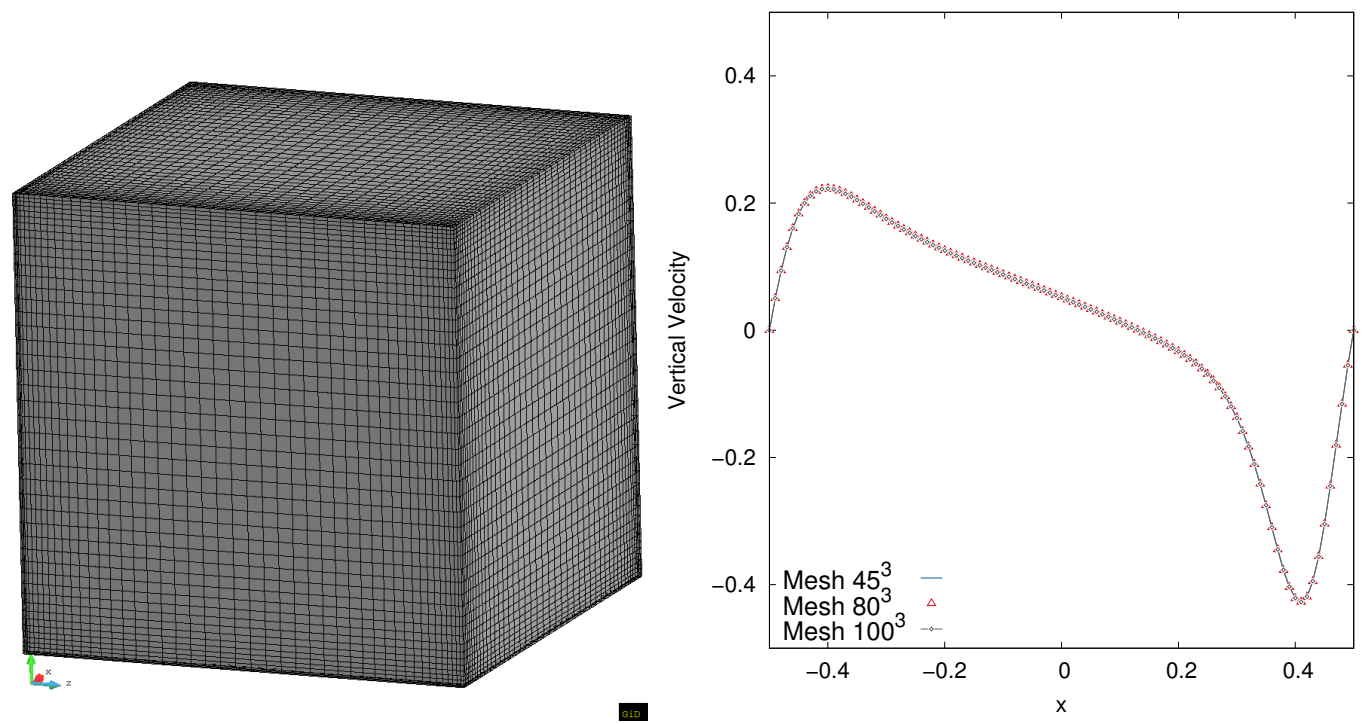

Figure 10: Mesh used for the cubic lid-driven cavity problem (left) and mesh convergence study (right).

To prove the capability of the proposed formulation to solve three-dimensional problems and to show that the method yields smooth and stable solutions for transient 3D flows, we solve the cubic lid-driven cavity flow for $\mathrm{Re}=1970$ using a very coarse mesh (with respect to papers devoted to the analysis of Hopf bifurcations) of $45 \times 45 \times 45$ hexahedral trilinear elements shown in Figure 10. For this problem we set the magnitude of the lid-driven velocity to 1 . The computational domain is defined as $\Omega=[-0.5,0.5] \times[-0.5,0.5] \times[-0.5,0.5]$. The kinematic viscosity is defined as $\nu=\frac{1}{\mathrm{Re}}$.

The time step value was set to $\delta t=0.1$. A convergence study based on capability of the mesh to capture the boundary layer associated to the vertical velocity in the horizontal middle cross line was done, corresponding to a steady state case close to the problem analyzed $(\mathrm{Re}=1900)$. In Figure 10 (right) it is seen that the three meshes analyzed $\left(45^{3}, 80^{3}\right.$ and $100^{3}$ nodes) yield almost overlapped results and, from this fact, the coarsest mesh was used for this numerical example, with the size distribution shown in Figure 10 (left).

In Figure 11, streamlines at three different time instants are presented to show the time-dependent nature of the solution. The zoom is located at the bottom left corner of the mid plane $y=0$. Note that the mesh used is very coarse to solve this type of problems; however, the dynamic term-by-term formulation is capable to capture the bifurcated solution.

Finally, in Figure 12 a representative snapshot is shown to present the flow pattern. In this figure the streamlines are plotted for a view of the plane $x z$.

\section{Conclusions}

In this work, we have proposed and analyzed an orthogonal dynamic term-by-term VMS method to solve incompressible flows. The orthogonal projections and the time tracking of the two velocity subscales, the convective one and the pressure gradient one, are the key ingredients. The main conclusions of the paper are summarized next: 

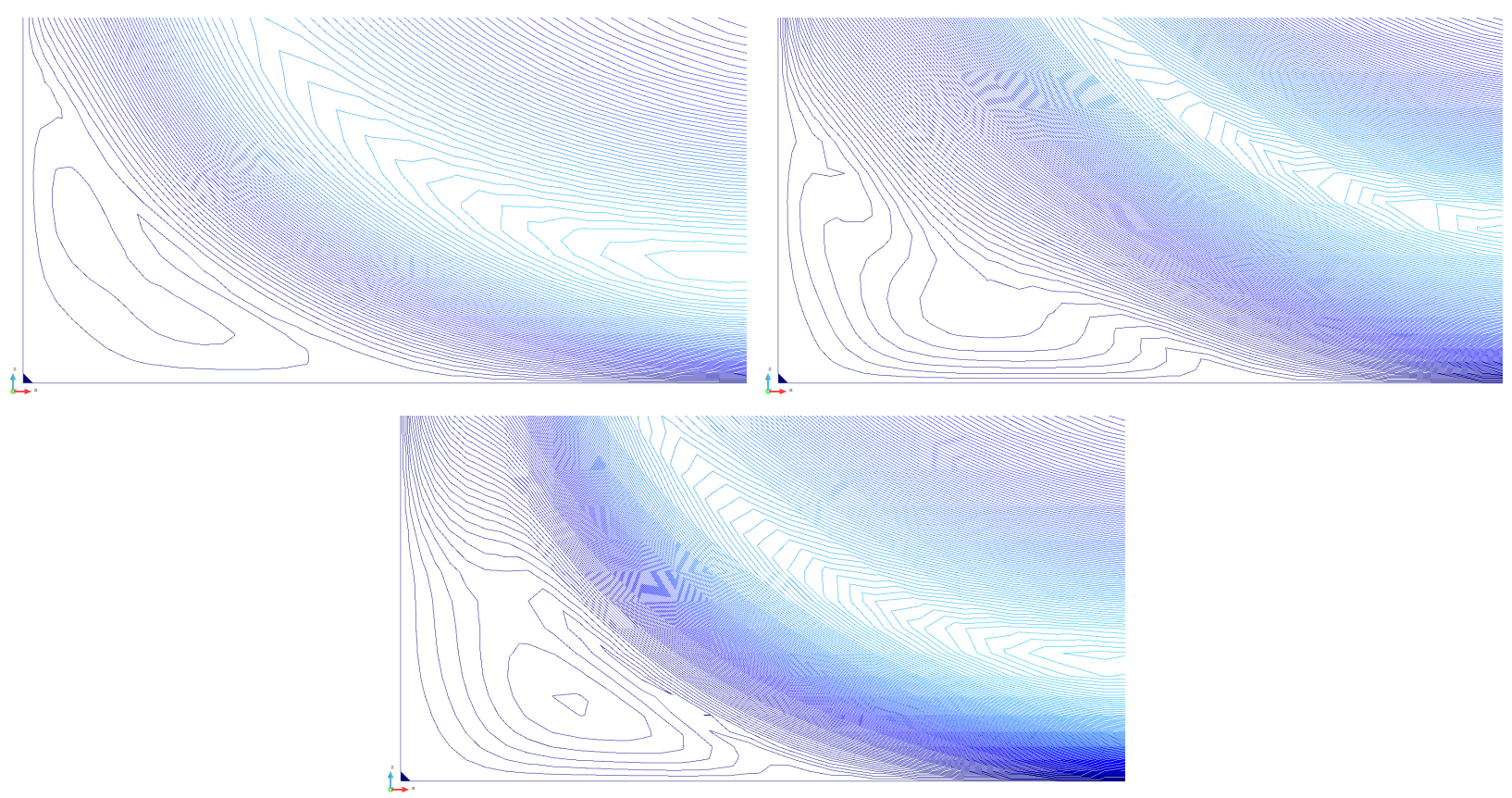

Figure 11: Vectors of velocity magnitude snapshot in the bottom left corner of the mid plane $y=0$ for different time instants (on top $t_{1}=15.6$ and $t_{2}=21.3$, and on bottom $t_{3}=26.25$ ).

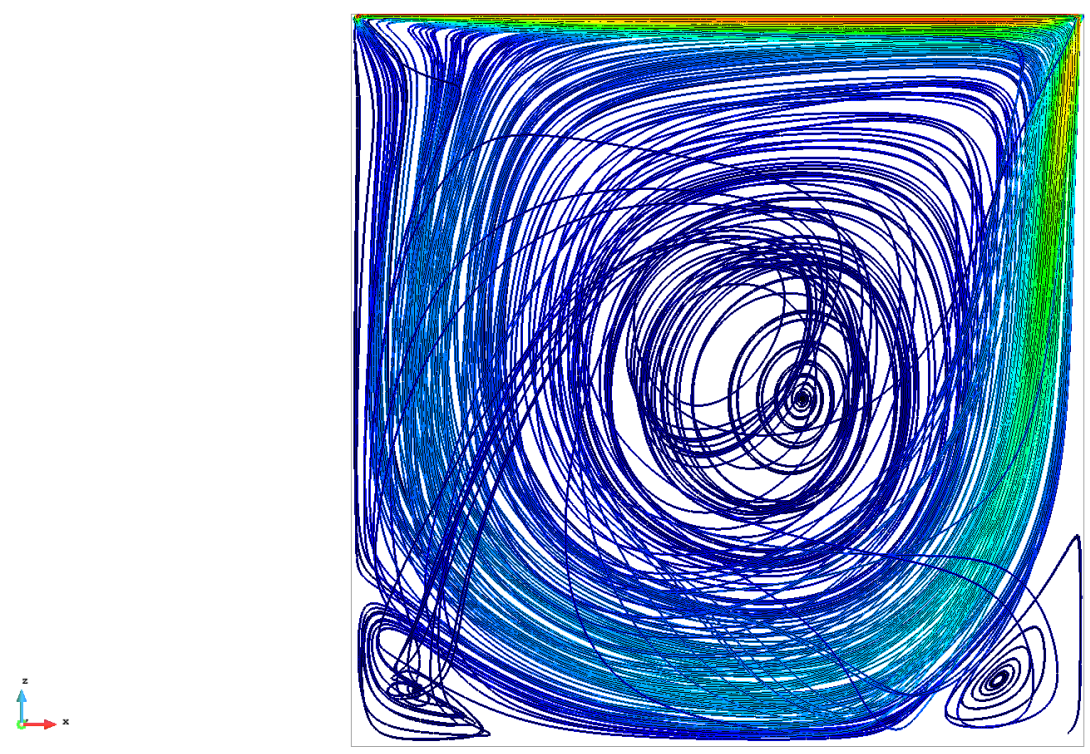

Figure 12: Velocity streamlines snapshot for the cubic lid-driven cavity problem. 
- The formulation has proven to be highly robust in all numerical tests, in laminar and in chaotic flows, in $2 D$ and $3 D$ examples, using linear and quadratic elements, and for structured and unstructured meshes.

- The orthogonal projections ensure that the consistency errors are of optimal order, a key point to use high order elements.

- The dissipative structure of the formulation has been analyzed and proved to be the correct one, opening the door to use the formulation in turbulent flows.

- The tracking of the subscales in time ensures stability even for anisotropic space-time discretizations, which has been proven mathematically (in a simplified case) and numerically up to the limit case $\delta t \approx \tau_{1, \min }$, both using linear and quadratic elements.

- The independency of the subscales that come from the the assumption of a quasi-selenoidal structure of the convective velocity subscale and the potential structure of the pressure gradient velocity subscale has been demonstrated numerically, even using quadratic elements.

\section{Acknowledgments}

This work has been partially funded by the Chilean Council for Scientic and Technological Research (CONICYT- FONDECYT 11160160). R. Codina acknowledges the support received through the ICREA Acàdemia Research Program, from the Catalan government.

\section{References}

[1] S. Badia and R. Codina, "On a multiscale approach to the transient Stokes problem: Dynamic subscales and anisotropic space-time discretization," Applied Mathematics and Computation, vol. 207, no. 2, pp. $415-433,2009$.

[2] T. J. Hughes, G. R. Feijóo, L. Mazzei, and J.-B. Quincy, "The variational multiscale method-a paradigm for computational mechanics," Computer Methods in Applied Mechanics and Engineering, vol. 166, no. 1, pp. 3 - 24, 1998. Advances in Stabilized Methods in Computational Mechanics.

[3] R. Codina, S. Badia, J. Baiges, and J. Principe, Variational Multiscale Methods in Computational Fluid Dynamics, in Encyclopedia of Computational Mechanics, pp. 1-28. John Wiley \& Sons, Ltd., 2017.

[4] R. Codina, "Stabilized finite element approximation of transient incompressible flows using orthogonal subscales," Computer Methods in Applied Mechanics and Engineering, vol. 191, no. 39, pp. 4295 - 4321, 2002.

[5] R. Codina, J. Principe, O. Guasch, and S. Badia, "Time dependent subscales in the stabilized finite element approximation of incompressible flow problems," Computer Methods in Applied Mechanics and Engineering, vol. 196, no. 21, pp. 2413 - 2430, 2007.

[6] J. Principe, R. Codina, and F. Henke, "The dissipative structure of variational multiscale methods for incompressible flows," Computer Methods in Applied Mechanics and Engineering, vol. 199, no. 13, pp. 791 - 801, 2010. Turbulence Modeling for Large Eddy Simulations. 
[7] O. Guasch and R. Codina, "Statistical behavior of the orthogonal subgrid scale stabilization terms in the finite element large eddy simulation of turbulent flows," Computer Methods in Applied Mechanics and Engineering, vol. 261-262, pp. 154 - 166, 2013.

[8] O. Colomés, S. Badia, R. Codina, and J. Principe, "Assessment of variational multiscale models for the large eddy simulation of turbulent incompressible flows," Computer Methods in Applied Mechanics and Engineering, vol. 285, pp. $32-63,2015$.

[9] F. Shakib and T. J. Hughes, "A new finite element formulation for computational fluid dynamics: IX. Fourier analysis of space-time Galerkin/least-squares algorithms," Computer Methods in Applied Mechanics and Engineering, vol. 87, no. 1, pp. 35 - 58, 1991.

[10] P. B. Bochev, M. D. Gunzburger, and R. B. Lehoucq, "On stabilized finite element methods for the Stokes problem in the small time step limit," International Journal for Numerical Methods in Fluids, vol. 53, no. 4, pp. 573-597, 2007.

[11] Y. Bazilevs, V. Calo, J. Cottrell, T. Hughes, A. Reali, and G. Scovazzi, "Variational multiscale residualbased turbulence modeling for large eddy simulation of incompressible flows," Computer Methods in Applied Mechanics and Engineering, vol. 197, no. 1, pp. 173 - 201, 2007.

[12] R. Codina, "Analysis of a stabilized finite element approximation of the Oseen equations using orthogonal subscales," Applied Numerical Mathematics, vol. 58, no. 3, pp. 264 - 283, 2008.

[13] E. Castillo and R. Codina, "Stabilized stress-velocity-pressure finite element formulations of the NavierStokes problem for fluids with non-linear viscosity," Computer Methods in Applied Mechanics and Engineering, vol. 279, pp. $554-578,2014$.

[14] E. Castillo and R. Codina, "Finite element approximation of the viscoelastic flow problem: A nonresidual based stabilized formulation," Computers \& Fluids, vol. 142, pp. 72 - 78, 2017. Selected papers of 18th International Conference on Finite Elements in Flow Problems.

[15] E. Castillo and R. Codina, "Variational multi-scale stabilized formulations for the stationary three-field incompressible viscoelastic flow problem," Computer Methods in Applied Mechanics and Engineering, vol. 279 , pp. $579-605,2014$.

[16] R. Codina, "A stabilized finite element method for generalized stationary incompressible flows," Computer Methods in Applied Mechanics and Engineering, vol. 190, no. 20, pp. 2681 - 2706, 2001.

[17] S. Badia, R. Codina, and J. Gutiérrez-Santacreu, "Long term stability estimates and existence of global attractors in a finite element approximation of the Navier-Stokes equations with numerical sub-grid scale modeling," SIAM Journal on Numerical Analysis, vol. 48, pp. 1013-1037, 2010.

[18] T. Chacón Rebollo, "A term by term stabilization algorithm for finite element solution of incompressible flow problems," Numerische Mathematik, vol. 79, pp. 283-319, Apr 1998.

[19] R. Codina, "Stabilization of incompressibility and convection through orthogonal sub-scales in finite element methods," Computer Methods in Applied Mechanics and Engineering, vol. 190, no. 13, pp. 1579 $-1599,2000$. 
[20] M. Braack and E. Burman, "Local projection stabilization for the Oseen problem and its interpretation as a variational multiscale method," SIAM Journal on Numerical Analysis, vol. 43, pp. 2544-2566, 2006.

[21] G. Matthies, P. Skrzypacz, and L. Tobiska, "A unified convergence analysis for local projection stabilisations applied to the Oseen problem," ESAIM: Mathematical Modelling and Numerical Analysis, vol. 41, pp. 713-742, 2007.

[22] P. Knobloch and G. Lube, "Local projection stabilization for advection-diffusion-reaction problems: Onelevel vs. two-level approach," Applied Numerical Mathematics, vol. 59, pp. 2891-2907, 2009.

[23] R. Codina, "On $h p$ convergence of stabilized finite element approximations of the convection-diffusion equation," SeMA Journal, vol. 75, pp. 591-606, 2018.

[24] E. Castillo and R. Codina, "First, second and third order fractional step methods for the three-field viscoelastic flow problem," Journal of Computational Physics, vol. 296, pp. 113 - 137, 2015.

[25] E. Burman, M. A. Fernández, and P. Hansbo, "Continuous interior penalty finite element method for Oseen's equations," SIAM Journal on Numerical Analysis, vol. 44, pp. 1248-1274, 2006.

[26] M. Braack and G. Lube, "Finite elements with local projection stabilization for incompressible flow problems," Journal of Computational Mathematics, vol. 27, pp. 116-147, 2009.

[27] T. Tezduyar and S. Sathe, "Stabilization parameters in SUPG and PSPG formulations," Journal of Computational and Applied Mechancis, vol. 4, no. 1, pp. 71 - 88, 2003.

[28] T. J. Hughes, G. Engel, L. Mazzei, and M. G. Larson, "The continuous Galerkin method is locally conservative," Journal of Computational Physics, vol. 163, no. 2, pp. 467 - 488, 2000.

[29] M. ten Eikelder and I. Akkerman, "Correct energy evolution of stabilized formulations: The relation between VMS, SUPG and GLS via dynamic orthogonal small-scales and isogeometric analysis. II: The incompressible Navier-Stokes equations," Computer Methods in Applied Mechanics and Engineering, To appear.

[30] R. Codina and O. Zienkiewicz, "CBS versus GLS stabilization of the incompressible Navier-Stokes equations and the role of the time step as stabilization parameter," Communications in Numerical Methods in Engineering, vol. 18, pp. 99-112, 2002.

[31] V. K. Patnana, R. P. Bharti, and R. P. Chhabra, "Two-dimensional unsteady flow of power-law fluids over a cylinder," Chemical Engineering Science, vol. 64, no. 12, pp. 2978 - 2999, 2009.

[32] R. Codina and J. Principe, "Dynamic subscales in the finite element approximation of thermally coupled incompressible flows," International Journal for Numerical Methods in Fluids, vol. 54, pp. 707-730, 2007.

[33] R. Codina, J. Principe, and M. Ávila, "Finite element approximation of turbulent thermally coupled incompressible flows with numerical sub-grid scale modelling," International Journal of Numerical Methods for Heat ES Fluid Flow, vol. 20, no. 5, pp. 492-516, 2010.

[34] Y. Feldman and A. Y. Gelfgat, "Oscillatory instability of a three-dimensional lid-driven flow in a cube," Physics of Fluids, vol. 22, no. 9, 2010, pp. 093602. 
[35] A. Liberzon, Y. Feldman, and A. Y. Gelfgat, "Experimental observation of the steady-oscillatory transition in a cubic lid-driven cavity," Physics of Fluids, vol. 23, no. 8, 2011, pp. 084106. 